\title{
Note on soft graviton theorem by KLT relation
}

\author{
Yi-Jian Du, ${ }^{1, a}$ Bo Feng, ${ }^{b, c}$ Chih-Hao $\mathbf{F u}^{c, d}$ and Yihong Wang ${ }^{e}$ \\ ${ }^{a}$ Department of Astronomy and Theoretical Physics, Lund University \\ SE-22362 Lund, Sweden \\ ${ }^{b}$ Zhejiang Institute of Modern Physics, Zhejiang University, \\ 38 Zheda Road Hangzhou, 310027 P.R China \\ ${ }^{c}$ Center of Mathematical Science, Zhejiang University, \\ 38 Zheda Road Hangzhou, 310027 P.R China \\ ${ }^{d}$ Department of Electrophysics, National Chiao Tung University \\ 1001 University Road, Hsinchu, Taiwan, R.O.C. \\ ${ }^{e}$ C. N. Yang Institute for Theoretical Physics, Stony Brook University, \\ Stony Brook, NY 11794, U.S.A. \\ E-mail: yijian.du@thep.lu.se, b.feng@cms.zju.edu.cn, \\ zhihao.fu@cms.zju.edu.cn, yihong.wang@stonybrook.edu
}

ABSTRACT: Recently, new soft graviton theorem proposed by Cachazo and Strominger has inspired a lot of works. In this note, we use the KLT-formula to investigate the theorem. We have shown how the soft behavior of color ordered Yang-Mills amplitudes can be combined with KLT relation to give the soft behavior of gravity amplitudes. As a byproduct, we find two nontrivial identities of the KLT momentum kernel must hold.

Keywords: Scattering Amplitudes, Strong Coupling Expansion, Duality in Gauge Field Theories, Gauge Symmetry

ArXiv EPRINT: 1408.4179

\footnotetext{
${ }^{1}$ On leave from Center for Field Theory and Particle Physics, Department of Physics, Fudan University, P.R China.
} 


\section{Contents}

1 Introduction 1

2 A review of KLT relation 3

3 Review of soft limits of gravity and Yang-Mills theory 4

$4 \quad$ KLT relation approach to the soft behavior of gravity amplitude $\quad 6$

$\begin{array}{lll}4.1 & \text { The leading order part } & 7\end{array}$

$\begin{array}{lll}4.2 & \text { The subleading order part } & 7\end{array}$

4.2.1 The sub-leading part from direct acting of $S_{G R}^{(1)} \quad 8$

4.2.2 The sub-leading order part from KLT relation 8

$\begin{array}{lll}4.2 .3 & \text { Comparing sub-leading parts } & 9\end{array}$

$\begin{array}{lll}4.3 & \text { The sub-sub-leading part from KLT relation } & 12\end{array}$

5 Examples $\quad \mathbf{1 2}$

5.1 The case $n=5 \quad 12$

$\begin{array}{ll}5.2 \text { The case } n=6 & 13\end{array}$

6 Conclusion $\quad 15$

$\begin{array}{ll}\text { A Example with } n=7 & 16\end{array}$

\section{Introduction}

Many scattering amplitudes were shown to have an universal soft behavior when the momentum of an external leg tends to zero. The soft limit can be traced back to the work [16]. In recent years, a new soft theorem for gravity amplitudes was studied in [7-9]. Using Britto-Cachazo-Feng-Witten (BCFW) recursion [10, 11], Cachazo and Strominger have proved the sub- and subsub- leading orders in the soft expansion [12], i.e., ${ }^{1}$

$$
M_{n+1}\left(\left\{\epsilon \lambda_{s}, \widetilde{\lambda}_{s}\right\}, 1, \ldots, n\right)=\left(\frac{1}{\epsilon^{3}} S_{G R}^{(0)}+\frac{1}{\epsilon^{2}} S_{G R}^{(1)}+\frac{1}{\epsilon} S_{G R}^{(2)}\right) M_{n}(1, \ldots, n)+\mathcal{O}\left(\epsilon^{0}\right) .
$$

The leading, subleading and subsubleading orders of soft factors are given by

$$
S_{G R}^{(0)}=\sum_{a=1}^{n} \frac{\varepsilon_{\mu \nu}^{s} p_{a}^{\mu} p_{a}^{\nu}}{p_{s} \cdot p_{a}}, \quad S_{G R}^{(1)}=-i \sum_{a=1}^{n} \frac{\varepsilon_{\mu \nu}^{s} p_{a}^{\mu}\left(p_{s, \rho} J_{a}^{\rho \nu}\right)}{p_{s} \cdot p_{a}}, \quad S_{G R}^{(2)}=\frac{-1}{2} \sum_{a=1}^{n} \frac{\varepsilon_{\mu \nu}^{s}\left(p_{s, \rho} J_{a}^{\rho \mu}\right)\left(p_{s, \sigma} J_{a}^{\sigma \nu}\right)}{p_{s} \cdot p_{a}}
$$

\footnotetext{
${ }^{1}$ The leading soft factor $S_{G R}^{(0)}$ is not corrected to all loop orders is shown in $[5,6,13]$ while the general subleading behavior of soft gluons and gravitons has also been discussed in [14-16].
} 
where the $\varepsilon_{\mu \nu}^{s}$ is the polarization of the soft graviton, $p_{i}$ are external momenta and $J^{\mu \nu}$ are angular momenta of external legs. Using the BCFW recursion relation, the soft limit of color-ordered tree-level Yang-Mills amplitudes was also studied in [17] and the result is given by

$$
A_{n+1}\left(\left\{\epsilon \lambda_{s}, \widetilde{\lambda}_{s}\right\}, 1, \ldots, n\right)=\left(\frac{1}{\epsilon^{2}} S_{Y M}^{(0)}+\frac{1}{\epsilon} S_{Y M}^{(1)}\right) A_{n}(1, \ldots, n)
$$

where the leading and subleading soft factors are given by

$$
S_{Y M}^{(0)}=\sum_{a \sim s} \frac{\varepsilon_{s} \cdot p_{a}}{p_{s} \cdot p_{a}}, \quad S_{Y M}^{(1)}=-i \sum_{a \sim s} \frac{\varepsilon_{s \nu} p_{s \mu} J_{a}^{\mu \nu}}{p_{s} \cdot p_{a}},
$$

with $\varepsilon_{s \nu}$ denoting the polarization of the soft gluon and $a \sim s$ meaning partial $a$ is next to soft particle $s$. Many related studies have been achieved including the soft limits from Poincare symmetry and gauge invariance [18, 19], Feynman diagram approach [20], conformal symmetry approach to the soft limits in Yang-Mills theory [21], the soft limit in arbitrary dimension [22-25], loop correction of the soft limit [26-28, 30], string-theory approach to the soft limit [29, 30] and ambitwistor string approach [31, 32].

In physics, it is very fruitful to study same thing from various angles because it will deepen our understanding and reveal many hidden relations. Now on-shell graviton scattering amplitudes can be calculated using many different ways, such as BCFW recursion relation, the double-copy formula [33], CHY formula [34, 35] and KLT formula [36] (and many more). Since the BCFW recursion relation and CHY formula have been successfully used in the study, in this note we will try to use the KLT formula to investigate the new soft graviton theorem.

Gravity amplitudes at tree level satisfy the famous Kawai-Lewellen-Tye (KLT) relation [36], with which, one can express the stripped tree-level gravity amplitudes $M_{n}$ (i.e., the momentum conservation $\delta^{4}\left(\sum p_{i}\right)$ has been moved away) in terms of products of tree-level color-ordered stripped Yang-Mills amplitudes $A_{n}$ and $\widetilde{A}_{n}$

$$
M_{n}(1,2, \ldots, n)=\sum_{\sigma, \rho} A_{n}(\sigma) \mathcal{S}[\sigma \mid \rho] \widetilde{A}_{n}(\rho),
$$

where $\mathcal{S}[\sigma \mid \rho]$ is called momentum kernel, which is a function of kinematic factors $s_{i j}=$ $2 p_{i} \cdot p_{j}$ and depends on the permutations $\sigma$ and $\rho .^{2}$ KLT relation was firstly proposed in string theory [36] and then was proved in field theory [37, 38] using BCFW recursion. One important feature should be emphasized is that KLT is relation between stripped amplitudes without imposing momentum conservation delta function.

Since KLT relation (1.5) connects gravity amplitudes to Yang-Mills amplitudes, it is natural to expect that the soft limit of gravity amplitudes can be derived from that of Yang-Mills amplitudes via KLT relation. In this work, we investigate this connection and its consequences. Although the KLT relation holds to general dimension, for simplicity we will focus on the pure 4D. We will show how the leading and sub-leading soft factors of

\footnotetext{
${ }^{2}$ In fact, the momentum kernel can be treated as the metric on the space of $(n-3)$ ! BCJ basis.
} 
gravity amplitudes can be reproduced by the leading and sub-leading soft factors of YangMills amplitudes as it should be. However, to reach such now well established fact, some nontrivial relations among changing matrix of $(n-3)$ ! BCJ-basis and momentum kernel $\mathcal{S}[\rho \mid \sigma]$ must be true. These nontrivial hidden identities are one of our main results.

The structure of this paper is following. In section 2, we provide a brief review of KLT relation. In section 3, we recall the soft limit for stripped amplitudes of gravity and Yang-Mills theory. In section 4, using results in section 3, we present the frame of the proof of the soft graviton soft theorem via KLT relation. In section 5, two examples have been given to demonstrate the frame in section 4 . In section 6 , we summarize our work with some future directions. In appendix A, we present another more complicated example.

\section{A review of KLT relation}

In this section, we provide a brief review of various formulations of KLT relation for gravity amplitudes (for more details, please refer [37, 38]). The most general formula [13] is given as

$$
\begin{aligned}
M_{n}(1,2, \ldots, n)= & (-1)^{n+1} \sum_{\sigma \in S_{n-3}} \sum_{\alpha \in S_{j-1}} \sum_{\beta \in S_{n-2-j}} A_{n}\left(1, \sigma_{2, j}, \sigma_{j+1, n-2}, n-1, n\right) \mathcal{S}\left[\alpha_{\sigma(2), \sigma(j)} \mid \sigma_{2, j}\right]_{p_{1}} \\
& \times \mathcal{S}\left[\sigma_{j+1, n-2} \mid \beta_{\sigma(j+1), \sigma(n-2)}\right]_{p_{n-1}} \widetilde{A}_{n}\left(\alpha_{\sigma(2), \sigma(j)}, 1, n-1, \beta_{\sigma(j+1), \sigma(n-2)}, n\right),
\end{aligned}
$$

where $A$ and $\widetilde{A}$ are two copies of color-ordered Yang-Mills amplitudes and the momentum kernel [37-39] is defined as

$$
\mathcal{S}\left[i_{1}, i_{2}, \ldots, i_{k} \mid j_{1}, j_{2}, \ldots, j_{k}\right]_{p_{1}}=\prod_{t=1}^{k}\left(s_{i_{t} 1}+\sum_{q>t}^{k} \theta\left(i_{t}, i_{q}\right) s_{i_{t} i_{q}}\right)
$$

where $p_{1}$ is the pivot and $\theta\left(i_{t}, i_{q}\right)$ is zero when pair $\left(i_{t}, i_{q}\right)$ has same ordering at both set $\mathcal{I}=\left\{i_{1}, i_{2}, \ldots, i_{k}\right\}, \mathcal{J}=\left\{j_{1}, j_{2}, \ldots, j_{k}\right\}$, otherwise it is one. ${ }^{3}$ In this definition, the set $\mathcal{J}=\left\{j_{1}, j_{2}, \ldots, j_{k}\right\}$ is the reference ordering set, i.e., this set provides the standard ordering. The set $\mathcal{I}=\left\{i_{1}, i_{2}, \ldots, i_{k}\right\}$ is the dynamical set which determines the dynamical factor by comparing with set $\mathcal{J}$. A few examples are the following:

$$
\mathcal{S}[2,3,4 \mid 2,4,3]_{p_{1}}=s_{21}\left(s_{31}+s_{34}\right) s_{41}, \quad \mathcal{S}[2,3,4 \mid 4,3,2]_{p_{1}}=\left(s_{21}+s_{23}+s_{24}\right)\left(s_{31}+s_{34}\right) s_{41} .
$$

Although it is not so obvious, the momentum kernel, in fact, contains all BCJ-relations by following identities

$$
0=\sum_{\alpha \in S_{n-2}} \mathcal{S}\left[\alpha\left(i_{2}, \ldots, i_{n-1}\right) \mid j_{2}, j_{3}, \ldots, j_{n-2}\right] A_{n}\left(n, \alpha\left(i_{2}, \ldots, i_{n-1}\right), 1\right), \forall j \in S_{n-2}
$$

Using (2.3) we can derive following relation

$$
\begin{aligned}
& \sum_{\alpha, \beta} \mathcal{S}\left[\alpha_{i_{2}, i_{j}} \mid i_{2}, \ldots, i_{j}\right]_{p_{1}} \mathcal{S}\left[i_{j+1}, \ldots, i_{n-2} \mid \beta_{i_{j+1}, i_{n-2}}\right]_{p_{n-1}} \widetilde{A}_{n}\left(\alpha_{i_{2}, i_{j}}, 1, n-1, \beta_{i_{j+1}, i_{n-2}}, n\right) \\
= & \sum_{\alpha^{\prime}, \beta^{\prime}} \mathcal{S}\left[\alpha_{i_{2}, i_{j-1}}^{\prime} \mid i_{2}, \ldots, i_{j-1}\right]_{p_{1}} \mathcal{S}\left[i_{j}, i_{j+1}, \ldots, i_{n-2} \mid \beta_{i_{j}, i_{n-2}}^{\prime}\right] \widetilde{A}_{n}\left(\alpha_{i_{2}, i_{j-1}}^{\prime}, 1, n-1, \beta_{i_{j}, i_{n-2}}^{\prime}, n\right),
\end{aligned}
$$

\footnotetext{
${ }^{3}$ The function $\mathcal{S}$ is nothing, but the $f$-function defined in [13] with more symmetric and improved expression
} 
Thus we can shift $j$ in (2.1) all the way to make the left- or right-hand part empty, i.e. we can choose $j=1$ or $j=n-2$. These special cases corresponds to the manifest $S_{n-3}$-symmetric form (2.5) and its dual form (2.6), which are given by

$M_{n}(1, \ldots, n)=(-)^{n+1} \sum_{\sigma, \widetilde{\sigma} \in S_{n-3}} A_{n}\left(1, \sigma_{2, n-2}, n-1, n\right) \mathcal{S}\left[\widetilde{\sigma}_{2, n-2} \mid \sigma_{2, n-2}\right]_{p_{1}} \widetilde{A}_{n}\left(n-1, n, \widetilde{\sigma}_{2, n-2}, 1\right)$.

and

$$
\begin{aligned}
& M_{n}(1, \ldots, n) \\
= & (-1)^{n+1} \sum_{\sigma, \widetilde{\sigma} \in S_{n-3}} A_{n}\left(1, \sigma_{2, n-2}, n-1, n\right) \mathcal{S}\left[\sigma_{2, n-2} \mid \widetilde{\sigma}_{2, n-2}\right]_{p_{n-1}} \widetilde{A}_{n}\left(1, n-1, \widetilde{\sigma}_{2, n-2}, n\right) .
\end{aligned}
$$

\section{Review of soft limits of gravity and Yang-Mills theory}

In this section, we review the soft behavior of gravity and Yang-Mills theory given in $[12,17]$. Since in KLT formula, amplitudes used are these stripped amplitudes, thus we will focus on the soft behaviors of these amplitudes.

We focus on the four dimensional case, thus we can use spinor variables. Under these variables, soft factors in (1.2) and (1.4) are given by [12] for gravity theory ${ }^{4}$

$$
\begin{aligned}
& S_{G R}^{(0)}=-\sum_{i=1}^{n} \frac{[s \mid i]\langle x \mid i\rangle\langle y \mid i\rangle}{\langle s \mid i\rangle\langle x \mid s\rangle\langle y \mid s\rangle}, \quad S_{G R}^{(1)}=-\frac{1}{2} \sum_{i=1}^{n} \frac{[s \mid i]}{\langle s \mid i\rangle}\left(\frac{\langle x \mid i\rangle}{\langle x \mid s\rangle}+\frac{\langle y \mid i\rangle}{\langle y \mid s\rangle}\right) \widetilde{\lambda}_{s}^{\dot{\alpha}} \frac{\partial}{\partial \widetilde{\lambda}_{i}^{\dot{\alpha}}} \\
& S_{G R}^{(2)}=-\frac{1}{2} \sum_{i=1}^{n} \frac{[s \mid i]}{\langle s \mid i\rangle} \widetilde{\lambda}_{s}^{\dot{\alpha}} \widetilde{\lambda}_{s}^{\dot{\beta}} \frac{\partial^{2}}{\partial \widetilde{\lambda}_{i}^{\dot{\alpha}} \widetilde{\lambda}_{i}^{\dot{\beta}}},
\end{aligned}
$$

where $x, y$ are two auxiliary spinors used to define the helicity of soft graviton

$$
\epsilon^{+2}=\left(\frac{\lambda_{x} \widetilde{\lambda}_{k}}{\langle x \mid k\rangle}\right)\left(\frac{\lambda_{y} \widetilde{\lambda}_{k}}{\langle y \mid k\rangle}\right)+\{x \leftrightarrow y\},
$$

and by [17] for Yang-Mills theory ${ }^{5}$

$$
S_{Y M}^{(0)}(n, s, 1, \ldots)=\frac{\langle n \mid 1\rangle}{\langle n \mid s\rangle\langle s \mid 1\rangle}, \quad S_{Y M}^{(1)}(n, s, 1, \ldots)=\frac{1}{\langle s \mid 1\rangle} \widetilde{\lambda}_{s} \frac{\partial}{\partial \widetilde{\lambda}_{1}}+\frac{1}{\langle n \mid s\rangle} \widetilde{\lambda}_{s} \frac{\partial}{\partial \widetilde{\lambda}_{n}} .
$$

To reach these expressions, we have used the fact that in $4 \mathrm{D}$, angular momentum can be written as spinor form

$$
J_{\mu \nu} \rightarrow-2 J_{\alpha \beta} \epsilon_{\dot{\alpha} \dot{\beta}}-2 \widetilde{J}_{\dot{\alpha} \dot{\beta}} \epsilon_{\alpha \beta}, \quad J_{\alpha \beta}=\frac{i}{2}\left(\lambda_{\alpha} \frac{\partial}{\partial \lambda^{\beta}}+\lambda_{\beta} \frac{\partial}{\partial \lambda^{\alpha}}\right), \quad \widetilde{J}_{\dot{\alpha} \dot{\beta}}=\frac{i}{2}\left(\widetilde{\lambda}_{\dot{\alpha}} \frac{\partial}{\partial \widetilde{\lambda}^{\dot{\beta}}}+\widetilde{\lambda}_{\dot{\beta}} \frac{\partial}{\partial \widetilde{\lambda}^{\dot{\alpha}}}\right) .
$$

We will explain the meaning of differential operators for stripped amplitudes shortly.

\footnotetext{
${ }^{4}$ It is worth to emphasize that here we have used the QCD convention, i.e., $2 p \cdot q=\langle p \mid q\rangle[q \mid p]$.

${ }^{5}$ We have assumed the color ordering is $(1, \ldots, n, s)$.
} 
For stripped amplitudes, we must impose momentum conservation from beginning. This can be done as given in [12]. Under the holomorphic soft limit which is defined as

$$
\lambda_{s} \rightarrow \epsilon \lambda_{s}, \quad \tilde{\lambda}_{s} \rightarrow \tilde{\lambda}_{s}
$$

momentum conservation $\sum_{i=1}^{n} k_{i}+\epsilon k_{s}=0$ can be used to solve two arbitrarily chosen anti-spinors $\widetilde{\lambda}_{i}, \widetilde{\lambda}_{j}$ as

$$
\widetilde{\lambda}_{i}=-\sum_{k \neq i, j} \frac{\langle j \mid k\rangle}{\langle j \mid i\rangle} \widetilde{\lambda}_{k}-\epsilon \frac{\langle j \mid s\rangle}{\langle j \mid i\rangle} \widetilde{\lambda}_{s}, \quad \widetilde{\lambda}_{j}=-\sum_{k \neq i, j} \frac{\langle i \mid k\rangle}{\langle i \mid j\rangle} \widetilde{\lambda}_{k}-\epsilon \frac{\langle i \mid s\rangle}{\langle i \mid j\rangle} \widetilde{\lambda}_{s}
$$

In other words, for stripped amplitudes, now the independent variables are $\lambda_{i}(i=1, \ldots, n)$, $\lambda_{s}, \widetilde{\lambda}_{s}$ and $\widetilde{\lambda}_{k}(k=1, \ldots, n$ and $k \neq i, j)$. With the fixed choice of pair $(i, j)$, when we use the BCFW recursion relation to discuss the soft behavior as was done in [27], for example, for an $(n+1)$-point color-ordered Yang-Mills amplitude $A\left(\left\{\epsilon \lambda_{s}, \widetilde{\lambda}_{s}\right\},\left\{\lambda_{1}, \widetilde{\lambda}_{1}\right\}, \ldots,\left\{\lambda_{n}, \widetilde{\lambda}_{n}\right\}\right)$ with $h_{s}=+1$, we will receive contributions to the singular part from the two-particle channel

$$
\left.A_{n+1}\left(\left\{\epsilon \lambda_{s}, \widetilde{\lambda}_{s}\right\}^{+}, 1, \ldots, n\right)\right|_{d i v}=\left.A_{3}\left(\widehat{s}^{+}, 1^{h_{1}},-\widehat{P}_{1 s}^{-h_{i}}\right) \frac{1}{P_{1 s}^{2}} A_{n}\left(\widehat{P}_{1 s}^{h_{i}}, \ldots, \widehat{n}\right)\right|_{d i v}
$$

under the $(s, n)$-shift

$$
\epsilon \lambda_{s}(z)=\epsilon \lambda_{s}+z \lambda_{n}, \quad \widetilde{\lambda}_{n}(z)=\widetilde{\lambda}_{n}-z \widetilde{\lambda}_{s} .
$$

It is easy to calculate the divergent part and we find

$$
\frac{-\langle n \mid 1\rangle}{\epsilon^{2}\langle n \mid s\rangle\langle s \mid 1\rangle} A_{n}\left(\left\{\lambda_{1}, \widetilde{\lambda}_{1}+\epsilon \frac{\langle n \mid s\rangle}{\langle n \mid 1\rangle} \widetilde{\lambda}_{s}\right\}^{h_{1}}, \ldots,\left\{\lambda_{i}, \widetilde{\lambda}_{i}(\epsilon)\right\}, \ldots\left\{\lambda_{j}, \widetilde{\lambda}_{j}(\epsilon)\right\}, \ldots,\left\{\lambda_{n}, \widetilde{\lambda}_{n}+\epsilon \frac{\langle 1 \mid s\rangle}{\langle 1 \mid n\rangle} \widetilde{\lambda}_{s}\right\}\right)
$$

where (3.6) must be used. A compact way to rewrite above expression is to assume $\widetilde{\lambda}_{i}, \widetilde{\lambda}_{j}$ to be independent first, so we have

$$
\begin{aligned}
& \frac{-\langle n \mid 1\rangle}{\epsilon^{2}\langle n \mid s\rangle\langle s \mid 1\rangle} \times \\
& \left\{\mathrm{e}^{\epsilon \frac{\langle n \mid s\rangle}{\langle n \mid 1\rangle} \widetilde{\lambda}_{s} \frac{\partial}{\partial \bar{\lambda}_{1}}-\epsilon \frac{\langle j \mid s\rangle}{\langle j \mid i\rangle} \widetilde{\lambda}_{s} \frac{\partial}{\partial \grave{\lambda}_{i}}-\epsilon \frac{\langle i \mid s\rangle}{\langle i \mid j\rangle} \widetilde{\lambda}_{s} \frac{\partial}{\partial \grave{\lambda}_{j}}+\frac{\epsilon\langle 1 \mid s\rangle}{\langle 1 \mid n\rangle} \widetilde{\lambda}_{s} \frac{\partial}{\partial \bar{\lambda}_{n}}} A_{n}\left(\left\{\lambda_{1}, \widetilde{\lambda}_{1}\right\}, \ldots,\left\{\lambda_{j}, \widetilde{\lambda}_{j}\right\}, \ldots,\left\{\lambda_{n}, \widetilde{\lambda}_{n}\right\}\right)\right\}
\end{aligned}
$$

Only after the action of $\frac{\partial}{\partial \widetilde{\lambda}_{i}}$ and $\frac{\partial}{\partial \widetilde{\lambda}_{j}}$, we can replace $\widetilde{\lambda}_{i}, \widetilde{\lambda}_{j}$ by (3.6) with $\epsilon=0$. However, if we insist to use (3.6) from beginning, $\widetilde{\lambda}_{i}, \widetilde{\lambda}_{j}$ will depend on $\widetilde{\lambda}_{1}, \widetilde{\lambda}_{n}$ thus the total derivative of $\frac{d}{d \widetilde{\lambda}_{1}}$ and $\frac{d}{d \widetilde{\lambda}_{n}}$ must be written as

$$
\begin{aligned}
& \frac{d}{d \widetilde{\lambda}_{1}}=\frac{\partial}{\partial \widetilde{\lambda}_{1}}+\frac{\partial}{\partial \widetilde{\lambda}_{i}}\left(-\frac{\langle j \mid 1\rangle}{\langle j \mid i\rangle}\right)+\frac{\partial}{\partial \widetilde{\lambda}_{j}}\left(-\frac{\langle i \mid 1\rangle}{\langle i \mid j\rangle}\right) \\
& \frac{d}{d \widetilde{\lambda}_{n}}=\frac{\partial}{\partial \widetilde{\lambda}_{n}}+\frac{\partial}{\partial \widetilde{\lambda}_{i}}\left(-\frac{\langle j \mid n\rangle}{\langle j \mid i\rangle}\right)+\frac{\partial}{\partial \widetilde{\lambda}_{j}}\left(-\frac{\langle i \mid n\rangle}{\langle i \mid j\rangle}\right) .
\end{aligned}
$$


Using above formula, it is easy to check that

$$
\frac{\langle n \mid s\rangle}{\langle n \mid 1\rangle} \widetilde{\lambda}_{s} \frac{d}{d \widetilde{\lambda}_{1}}+\frac{\langle 1 \mid s\rangle}{\langle 1 \mid n\rangle} \widetilde{\lambda}_{s} \frac{d}{d \widetilde{\lambda}_{n}}=\frac{\langle n \mid s\rangle}{\langle n \mid 1\rangle} \widetilde{\lambda}_{s} \frac{\partial}{\partial \widetilde{\lambda}_{1}}+\frac{\langle 1 \mid s\rangle}{\langle 1 \mid n\rangle} \widetilde{\lambda}_{s} \frac{\partial}{\partial \widetilde{\lambda}_{n}}-\frac{\langle j \mid s\rangle}{\langle j \mid i\rangle} \widetilde{\lambda}_{s} \frac{\partial}{\partial \widetilde{\lambda}_{i}}-\frac{\langle i \mid s\rangle}{\langle i \mid j\rangle} \widetilde{\lambda}_{s} \frac{\partial}{\partial \widetilde{\lambda}_{j}},
$$

thus (3.10) becomes

$$
\frac{-\langle n \mid 1\rangle}{\epsilon^{2}\langle n \mid s\rangle\langle s \mid 1\rangle}\left\{\mathbf{e}^{\frac{\langle n \mid s\rangle}{\langle n \mid 1\rangle} \widetilde{\lambda}_{s} \frac{d}{d \tilde{\lambda}_{1}}+\frac{\langle 1 \mid s\rangle}{\langle 1 \mid n\rangle} \widetilde{\lambda}_{s} \frac{d}{d \tilde{\lambda}_{n}}} A_{n}\left(\left\{\lambda_{1}, \widetilde{\lambda}_{1}\right\}, \ldots,\left\{\lambda_{j}, \widetilde{\lambda}_{j}\right\}, \ldots,\left\{\lambda_{n}, \widetilde{\lambda}_{n}\right\}\right)\right\}
$$

Having this new understanding, the meaning of soft factors in (3.1) and (3.3) becomes clear: while there are no variables $\widetilde{\lambda}_{i}, \widetilde{\lambda}_{j}$ anymore in stripped amplitudes, all partial derivatives should be considered as a kind of "total derivative" in the sense of (3.11).

\section{KLT relation approach to the soft behavior of gravity amplitude}

Having above preparations, now we study the soft behavior of stripped gravity amplitudes using the soft behavior of stripped Yang-Mills amplitudes as input through KLT relation. The total symmetry among the $n$-particles of gravity amplitudes allows us to choose any leg to be soft leg. We take $p_{1}$ to be soft and solve $n-1, n$ as

$\widetilde{\lambda}_{n-1}=-\sum_{k=2}^{n-2} \frac{\langle n \mid k\rangle}{\langle n \mid n-1\rangle} \widetilde{\lambda}_{k}-\epsilon \frac{\langle n \mid 1\rangle}{\langle n \mid n-1\rangle} \widetilde{\lambda}_{1}, \quad \widetilde{\lambda}_{n}=-\sum_{k=2}^{n-2} \frac{\langle n-1 \mid k\rangle}{\langle n-1 \mid n\rangle} \widetilde{\lambda}_{k}-\epsilon \frac{\langle n-1 \mid 1\rangle}{\langle n-1 \mid n\rangle} \widetilde{\lambda}_{1}$.

The choice of KLT formula: in section 2, we have reviewed various formulations of KLT relation. To make the discussion simpler, we should start with proper choice of KLT formula. Since the leading contribution from two gluon amplitudes is the order $\frac{1}{\epsilon^{2}} \times \frac{1}{\epsilon^{2}}$ while the leading contribution of graviton amplitude is $\frac{1}{\epsilon^{3}}$, we are better to have manifest $\epsilon$-factor from kernel part. Furthermore, since we have solved $\widetilde{\lambda}_{n-1}, \widetilde{\lambda}_{n}$ in (4.1), it is more convenient to have formula as less related to $p_{n-1}, p_{n}$ as possible. Taking these things into consideration, we use the general formula given by (2.1) with $j=2$

$$
M_{n}=(-1)^{n+1} \sum_{t=2}^{n-2} \sum_{\sigma, \beta \in S_{n-4}} A_{n}(1, t, \sigma, n-1, n) \mathcal{S}[t \mid t]_{p_{1}} \mathcal{S}[\sigma \mid \beta]_{p_{n-1}} \widetilde{A}_{n}(t, 1, n-1, \beta, n)
$$

In this form, $\mathcal{S}[t \mid t]_{p_{1}} \rightarrow \epsilon s_{1 t}$, while the expansion of the other kernel $\mathcal{S}[\sigma \mid \beta]_{p_{n-1}}$ can be written as $^{6}$

$$
\mathbf{e}^{+\epsilon \frac{\langle n \mid 1\rangle}{\langle n \mid t\rangle} \widetilde{\lambda}_{1} \frac{d}{d \widetilde{\lambda}_{t}}} \mathcal{S}[\sigma \mid \beta]_{p_{n-1}} .
$$

\footnotetext{
${ }^{6}$ From the definition of kernel, the $\epsilon$-expansion should be given by $\mathbf{e}^{-\epsilon \frac{\langle n \mid 1\rangle}{\langle n \mid n-1\rangle} \widetilde{\lambda}_{1} \mid \frac{\partial}{\partial \tilde{\lambda}_{n-1}}} \mathcal{S}[\sigma \mid \beta]_{p_{n-1}}$. However, noticing that

$$
\widetilde{\lambda}_{1} \frac{d}{d \widetilde{\lambda}_{t}} \mathcal{S}[\sigma \mid \beta]_{p_{n-1}}=\widetilde{\lambda}_{1}\left(-\frac{\langle n \mid t\rangle}{\langle n \mid n-1\rangle}\right) \frac{\partial}{\partial \widetilde{\lambda}_{n-1}} \mathcal{S}[\sigma \mid \beta]_{p_{n-1}}
$$
}

where we have used the fact that $\widetilde{\lambda}_{s} \frac{d}{d \widetilde{\lambda}_{t}} \mathcal{S}[\sigma \mid \beta]_{p_{n-1}}$ does not contain momentum $p_{t}$, we obtain (4.3). 
For convenience, we use (3.3) to write down the singular soft limit of two stripped amplitudes in (4.2) as

$$
\begin{aligned}
A_{n}^{(n-1, n)}(1, t, \sigma, n-1, n) \rightarrow & \frac{1}{\epsilon^{2}} \frac{\langle n \mid t\rangle}{\langle n \mid 1\rangle\langle 1 \mid t\rangle} A_{n-1}^{(n-1, n)}(t, \sigma, n-1, n) \\
& +\frac{1}{\epsilon} \frac{\langle n \mid t\rangle}{\langle n \mid 1\rangle\langle 1 \mid t\rangle}\left(\frac{\langle n \mid 1\rangle}{\langle n \mid t\rangle} \widetilde{\lambda}_{1} \frac{d}{\widetilde{\lambda}_{t}}+\frac{\langle t \mid 1\rangle}{\langle t \mid n\rangle} \widetilde{\lambda}_{1} \frac{d}{d \widetilde{\lambda}_{n}}\right) A_{n-1}(t, \sigma, n-1, n),(t, \\
\widetilde{A}_{n}^{(n-1, n)}(t, 1, n-1, \beta, n) \rightarrow & \frac{1}{\epsilon^{2}} \frac{\langle t \mid n-1\rangle}{\langle t \mid 1\rangle\langle 1 \mid n-1\rangle} \widetilde{A}_{n}(t, n-1, \beta, n) \\
+ & \frac{1}{\epsilon} \frac{\langle t \mid n-1\rangle}{\langle t \mid 1\rangle\langle 1 \mid n-1\rangle}\left(\frac{\langle t \mid 1\rangle}{\langle t \mid n-1\rangle} \widetilde{\lambda}_{1} \frac{d}{d \widetilde{\lambda}_{n-1}}+\frac{\langle n-1 \mid 1\rangle}{\langle n-1 \mid t\rangle} \widetilde{\lambda}_{1} \frac{d}{d \widetilde{\lambda}_{t}}\right) \widetilde{A}_{n}(t, n-1, \beta, n) .
\end{aligned}
$$

In the remainder of this section, we discuss the soft behavior of gravity amplitudes by KLT relations order by order.

\subsection{The leading order part}

Substituting the leading part of color-ordered Yang-Mills amplitudes $A, \widetilde{A}$ (given by $\frac{1}{\epsilon^{2}}$ terms of (4.4), (4.5)) as well as the leading part of momentum kernel $\mathcal{S}$ (given by the $\epsilon$ term of $\mathcal{S}[t \mid t]_{p_{1}} \mathcal{S}[\sigma \mid \beta]_{p_{n-1}}$ ) into the KLT expression (4.2), we get the leading part of gravity amplitude under soft limit

$$
\begin{aligned}
M_{n}= & (-1)^{n+1} \sum_{t=2}^{n-2} \sum_{\sigma, \beta \in S_{n-4}} \frac{1}{\epsilon^{2}} \frac{\langle n \mid t\rangle}{\langle n \mid 1\rangle\langle 1 \mid t\rangle} A_{n-1}^{(n-1, n)}(t, \sigma, n-1, n) \epsilon s_{1 t} \mathcal{S}[\sigma \mid \beta]_{p_{n-1}^{\epsilon \rightarrow 0}} \\
& \frac{1}{\epsilon^{2}} \frac{\langle t \mid n-1\rangle}{\langle t \mid 1\rangle\langle 1 \mid n-1\rangle} \widetilde{A}_{n}^{(n-1, n)}(t, n-1, \beta, n) \\
= & \frac{1}{\epsilon^{3}}(-1)^{n+1} \sum_{t=2}^{n-2} \frac{[t \mid 1]}{\langle t \mid 1\rangle} \frac{\langle n \mid t\rangle}{\langle n \mid 1\rangle} \frac{\langle n-1 \mid t\rangle}{\langle n-1 \mid 1\rangle} \times \\
& \sum_{\sigma, \beta \in S_{n-4}} A_{n-1}^{(n-1, n)}(t, \sigma, n-1, n) \mathcal{S}[\sigma \mid \beta]_{p_{n-1}^{\epsilon \rightarrow 0}} \widetilde{A}_{n}^{(n-1, n)}(t, n-1, \beta, n) \\
= & \frac{1}{\epsilon^{3}}(-) \sum_{t=2}^{n-2} \frac{[t \mid 1]}{\langle t \mid 1\rangle} \frac{\langle n \mid t\rangle}{\langle n \mid 1\rangle} \frac{\langle n-1 \mid t\rangle}{\langle n-1 \mid 1\rangle} M_{n-1}(2, \ldots, n) \\
= & \frac{1}{\epsilon^{3}} S_{G R}^{(0)} M_{n-1}(2, \ldots, n)
\end{aligned}
$$

where, on the third line, we have used the $S_{n-3}$-symmetric KLT relation (2.6) for $(n-1)$ point amplitudes. The soft factor of gravity is nothing but the $S_{G R}^{(0)}$ defined in (3.1) with $x=n$ and $y=n-1$.

\subsection{The subleading order part}

Now let us study the subleading order of stripped gravity amplitudes under the soft limit. We will do it in three steps. In the first step, we act the $S_{G R}^{(1)}$ defined in (3.1) on the 
KLT expressions (2.6) of $(n-1)$-point gravity amplitudes directly. In the second step, we collect contributions of the subleading part from color ordered Yang-Mills amplitudes and momentum kernel in (4.2). Finally, we compare the two expressions from first two steps to prove (check) the subleading order soft factor $S_{G R}^{(1)}$ of gravity amplitude.

\subsubsection{The sub-leading part from direct acting of $S_{G R}^{(1)}$}

We use the subleading soft factor given by (3.1)

$$
S_{G R,(n-1) n}^{(1)}=-\sum_{i=2}^{n} \frac{[1 \mid i]}{\langle 1 \mid i\rangle} \frac{\langle n-1 \mid i\rangle}{\langle n-1 \mid 1\rangle} \widetilde{\lambda}_{1} \frac{d}{d \widetilde{\lambda}_{i}}=-\sum_{i=2}^{n-2} \frac{[1 \mid i]}{\langle 1 \mid i\rangle} \frac{\langle n-1 \mid i\rangle}{\langle n-1 \mid 1\rangle} \widetilde{\lambda}_{1} \frac{d}{d \widetilde{\lambda}_{i}}
$$

where we have taken the gauge choice $x=y=n-1$, thus $\frac{d}{d \widetilde{\lambda}_{n-1}}=\frac{d}{d \widetilde{\lambda}_{n}}=0$. When acting it with the form (4.7) on $M_{n-1}$, for each $i$, we take different representation of $M_{n-1},{ }^{7}$ i.e.,

$$
\begin{aligned}
& S_{G R,(n-1) n}^{(1)} M_{n-1}(2, \ldots, n)=-\sum_{i=2}^{n-2} \frac{[1 \mid i]}{\langle 1 \mid i\rangle} \frac{\langle n-1 \mid i\rangle}{\langle n-1 \mid 1\rangle} \widetilde{\lambda}_{1} \frac{d}{d \widetilde{\lambda}_{i}} M_{n-1}(2, \ldots, n) \\
= & -\sum_{i=2}^{n-2} \frac{[1 \mid i]}{\langle 1 \mid i\rangle} \frac{\langle n-1 \mid i\rangle}{\langle n-1 \mid 1\rangle} \widetilde{\lambda}_{1} \frac{d}{d \widetilde{\lambda}_{i}}\left[(-1)^{n} \sum_{\sigma, \beta \in S_{n-4}} A_{n-1}(i, \sigma, n-1, n) \mathcal{S}[\sigma \mid \beta]_{p_{n-1}} \widetilde{A}_{n-1}(i, n-1, \beta, n)\right] \\
= & (-)^{n+1} \sum_{i=2}^{n-2} \sum_{\sigma, \beta \in S_{n-4}} \frac{[1 \mid i]}{\langle 1 \mid i\rangle} \frac{\langle n-1 \mid i\rangle}{\langle n-1 \mid 1\rangle} A_{n-1}(i, \sigma, n-1, n)\left(\widetilde{\lambda}_{1} \frac{d}{d \widetilde{\lambda}_{i}} \mathcal{S}[\sigma \mid \beta]_{p_{n-1}}\right) \widetilde{A}_{n-1}(i, n-1, \beta, n) \\
& +(-)^{n+1} \sum_{i=2}^{n-2} \sum_{\sigma, \beta \in S_{n-4}} \frac{[1 \mid i]}{\langle 1 \mid i\rangle} \frac{\langle n-1 \mid i\rangle}{\langle n-1 \mid 1\rangle}\left(\widetilde{\lambda}_{1} \frac{d}{d \widetilde{\lambda}_{i}} A_{n-1}(i, \sigma, n-1, n)\right) \mathcal{S}[\sigma \mid \beta]_{p_{n-1}} \widetilde{A}_{n-1}(i, n-1, \beta, n) \\
& +(-)^{n+1} \sum_{i=2}^{n-2} \sum_{\sigma, \beta \in S_{n-4}} \frac{[1 \mid i]}{\langle 1 \mid i\rangle} \frac{\langle n-1 \mid i\rangle}{\langle n-1 \mid 1\rangle} A_{n-1}(i, \sigma, n-1, n) \mathcal{S}[\sigma \mid \beta]_{p_{n-1}}\left(\widetilde{\lambda}_{1} \frac{d}{d \widetilde{\lambda}_{i}} \widetilde{A}_{n-1}(i, n-1, \beta, n)\right) .
\end{aligned}
$$

\subsubsection{The sub-leading order part from KLT relation}

Now we collect the contributions of the subleading part from the KLT relation (4.2). There are three contributions at this order. The first term is to take kernel to second order of $\epsilon$, while $A, \widetilde{A}$ are the first order (see (4.4) and (4.5)). This part is given by

$$
\begin{aligned}
T_{1}= & (-1)^{n+1} \sum_{t=2}^{n-2} \sum_{\sigma, \beta \in S_{n-4}} \frac{[1 \mid t]}{\langle 1 \mid t\rangle} \frac{\langle n \mid t\rangle\langle n-1 \mid t\rangle}{\langle n \mid 1\rangle\langle n-1 \mid 1\rangle} A_{n-1}(t, \sigma, n-1, n)\left(\frac{\langle n \mid 1\rangle}{\langle n \mid t\rangle} \widetilde{\lambda}_{1} \frac{d}{d \widetilde{\lambda}_{t}} \mathcal{S}[\sigma \mid \beta]_{p_{n-1}}\right) \times \\
& \widetilde{A}_{n-1}(t, n-1, \beta, n) \\
= & (-1)^{n+1} \sum_{t=2}^{n-2} \sum_{\sigma, \beta \in S_{n-4}} \frac{[1 \mid t]}{\langle 1 \mid t\rangle} \frac{\langle n-1 \mid t\rangle}{\langle n-1 \mid 1\rangle} A_{n-1}(t, \sigma, n-1, n)\left(\widetilde{\lambda}_{1} \frac{d}{d \widetilde{\lambda}_{t}} \mathcal{S}[\sigma \mid \beta]_{p_{n-1}}\right) \widetilde{A}_{n-1}(t, n-1, \beta, n)
\end{aligned}
$$

\footnotetext{
${ }^{7}$ It is worth to notice that although as a whole, we have the freedom to chose $x, y$ for $S_{G R,(n-1) n}^{(1)}$, when we act it for different $i$ and different part $A, \widetilde{A}$ in (4.8), we need to stick to a particular gauge choice.
} 
For the second term, we keep the leading order of kernel and $\widetilde{A}$ while taking the subleading order of $A$, thus we have

$$
\begin{aligned}
T_{2}= & (-1)^{n+1} \sum_{t=2}^{n-2} \sum_{\sigma, \beta \in S_{n-4}} \frac{[1 \mid t]}{\langle 1 \mid t\rangle} \frac{\langle n \mid t\rangle\langle n-1 \mid t\rangle}{\langle n \mid 1\rangle\langle n-1 \mid 1\rangle} \\
& \times\left[\left(\frac{\langle n \mid 1\rangle}{\langle n \mid t\rangle} \widetilde{\lambda}_{1} \frac{d}{d \widetilde{\lambda}_{t}}+\frac{\langle t \mid 1\rangle}{\langle t \mid n\rangle} \widetilde{\lambda}_{1} \frac{d}{d \widetilde{\lambda}_{n}}\right) A_{n-1}(t, \sigma, n-1, n)\right] \mathcal{S}[\sigma \mid \beta]_{p_{n-1}} \widetilde{A}_{n}(t, n-1, \beta, n) \\
= & (-1)^{n+1} \sum_{t=2}^{n-2} \sum_{\sigma, \beta \in S_{n-4}} \frac{[1 \mid t]}{\langle 1 \mid t\rangle} \frac{\langle n-1 \mid t\rangle}{\langle n-1 \mid 1\rangle}\left(\widetilde{\lambda}_{1} \frac{d}{d \widetilde{\lambda}_{t}} A_{n-1}(t, \sigma, n-1, n)\right) \mathcal{S}[\sigma \mid \beta]_{p_{n-1}} \times \\
& \left(\widetilde{A}_{n}(t, n-1, \beta, n)\right),
\end{aligned}
$$

where we have used the fact that $\frac{d}{d \widetilde{\lambda}_{n}} A_{n-1}(t, \sigma, n-1, n)=0$

For the third term, we keep the leading order of kernel and $A$ while take the subleading order of $\widetilde{A}$, thus we have

$$
\begin{aligned}
T_{3}= & (-1)^{n+1} \sum_{t=2}^{n-2} \sum_{\sigma, \beta \in S_{n-4}} \frac{[1 \mid t]}{\langle 1 \mid t\rangle} \frac{\langle n \mid t\rangle\langle n-1 \mid t\rangle}{\langle n \mid 1\rangle\langle n-1 \mid 1\rangle} A_{n-1}(t, \sigma, n-1, n) \mathcal{S}[\sigma \mid \beta]_{p_{n-1}} \\
& \left(\left\{\frac{\langle t \mid 1\rangle}{\langle t \mid n-1\rangle} \widetilde{\lambda}_{1} \frac{d}{d \widetilde{\lambda}_{n-1}}+\frac{\langle n-1 \mid 1\rangle}{\langle n-1 \mid t\rangle} \widetilde{\lambda}_{1} \frac{d}{d \widetilde{\lambda}_{t}}\right\} \widetilde{A}_{n}^{(n-1, n)}(t, n-1, \beta, n)\right) \\
= & (-1)^{n+1} \sum_{t=2}^{n-2} \sum_{\sigma, \beta \in S_{n-4}} \frac{[1 \mid t]}{\langle 1 \mid t\rangle} \frac{\langle n \mid t\rangle}{\langle n \mid 1\rangle} A_{n-1}(t, \sigma, n-1, n) \mathcal{S}[\sigma \mid \beta]_{p_{n-1}}\left(\widetilde{\lambda}_{1} \frac{d}{d \widetilde{\lambda}_{t}} \widetilde{A}_{n}^{(n-1, n)}(t, n-1, \beta, n)\right)
\end{aligned}
$$

where again we have used the fact $\widetilde{\lambda}_{1} \frac{d}{d \widetilde{\lambda}_{n-1}} \widetilde{A}_{n}^{(n-1, n)}(t, n-1, \beta, n)=0$.

\subsubsection{Comparing sub-leading parts}

Now we compare (4.8) with $T_{1}, T_{2}, T_{3}$. It is easy to see when we identify $i=t$, we have

$$
\begin{aligned}
\Delta= & S_{G R,(n-1) n}^{(1)} M_{n-1}(2, \ldots, n)-T_{1}-T_{2}-T_{3} \\
= & (-1)^{n+1} \sum_{t=2}^{n-2} \sum_{\sigma, \beta \in S_{n-4}} \frac{[1 \mid t]}{\langle 1 \mid t\rangle}\left(\frac{\langle n-1 \mid t\rangle}{\langle n-1 \mid 1\rangle}-\frac{\langle n \mid t\rangle}{\langle n \mid 1\rangle}\right) A_{n-1}(t, \sigma, n-1, n) \mathcal{S}[\sigma \mid \beta]_{p_{n-1}} \times \\
& \left(\widetilde{\lambda}_{1} \frac{d}{d \widetilde{\lambda}_{t}} \widetilde{A}_{n}^{(n-1, n)}(t, n-1, \beta, n)\right) \\
= & (-1)^{n+1} \sum_{t=2}^{n-2} \sum_{\sigma, \beta \in S_{n-4}} \frac{[1 \mid t]\langle n \mid n-1\rangle}{\langle n-1 \mid 1\rangle\langle n \mid 1\rangle} A_{n-1}(t, \sigma, n-1, n) \mathcal{S}[\sigma \mid \beta]_{p_{n-1}} \times \\
& \left(\widetilde{\lambda}_{1} \frac{d}{d \widetilde{\lambda}_{t}} \widetilde{A}_{n}^{(n-1, n)}(t, n-1, \beta, n)\right) \\
= & (-1)^{n+1} \sum_{t=2}^{n-2} \sum_{\sigma, \beta \in S_{n-4}} \frac{\widetilde{\lambda}_{1}^{\dot{\alpha}} \widetilde{\lambda}_{1}^{\dot{\beta}}\langle n \mid n-1\rangle}{\langle n-1 \mid 1\rangle\langle n \mid 1\rangle} A_{n-1}(t, \sigma, n-1, n) \mathcal{S}[\sigma \mid \beta]_{p_{n-1}} \times \\
& \left(\widetilde{\lambda}_{t, \dot{\alpha}} \frac{d}{d \widetilde{\lambda}_{t}^{\dot{\beta}}} \widetilde{A}_{n}^{(n-1, n)}(t, n-1, \beta, n)\right)
\end{aligned}
$$




$$
\begin{aligned}
= & (-1)^{n+1} \sum_{t=2}^{n-2} \sum_{\sigma, \beta \in S_{n-4}} \frac{\widetilde{\lambda}_{1}^{\dot{\alpha}} \widetilde{\lambda}_{1}^{\dot{\beta}}\langle n \mid n-1\rangle}{\langle n-1 \mid 1\rangle\langle n \mid 1\rangle} A_{n-1}(t, \sigma, n-1, n) \mathcal{S}[\sigma \mid \beta]_{p_{n-1}}(-i) \times \\
& \left(J_{t, \dot{\alpha} \dot{\beta}} \widetilde{A}_{n}(t, n-1, \beta, n)\right)
\end{aligned}
$$

It is obviously that to prove (or check) the subleading soft factor $S_{G R,(n-1) n}^{(1)}$, we need to prove (or check) $\Delta=0$. Before going to the detail, let us notice that in (4.12) only the anti-spinor part of angular momentum $J_{t, \dot{\alpha} \dot{\beta}}$ appears.

Now we present the idea of proof. In (4.12), for each $t$, we have used different BCJbasis for color ordered partial amplitudes. Thus the first step is to translate various basis into a standard basis. In other words, we should do following transformation

$$
\begin{aligned}
& A_{n-1}\left(t, \sigma_{t}, n-1, n\right)=\sum_{\sigma_{\tilde{t} \in S_{n-4}}} A_{n-1}\left(\widetilde{t}, \sigma_{\widetilde{t}}, n-1, n\right) \mathcal{D}\left[\widetilde{t}, \sigma_{\widetilde{t}}, n-1, n \mid t, \sigma_{t}, n-1, n\right] \\
& \widetilde{A}_{n-1}\left(t, n-1, \beta_{t}, n\right)=\sum_{\beta_{\tilde{t}} \in S_{n-4}} \mathcal{C}\left[t, n-1, \beta_{t}, n \mid \widetilde{t}, n-1, \beta_{\widetilde{t}}, n\right] \widetilde{A}_{n-1}\left(\widetilde{t}, n-1, \beta_{\widetilde{t}}, n\right) .
\end{aligned}
$$

where we have used the $\sigma_{t}$ to denote the permutations of $n$-4-elements after deleting particles $1, n, n-1, t$. Inserting above transformation into the extra term (4.12), when we choose e.g., $\widetilde{t}=2$ in above equations, we obtain

$$
\begin{aligned}
& (-1)^{n+1} \Delta \\
& =(-i) \sum_{\sigma_{2}, \beta_{2} \in S_{n-4}} \frac{\widetilde{\lambda}_{1}^{\dot{\alpha}} \widetilde{\lambda}_{1}^{\dot{\beta}}\langle n \mid n-1\rangle}{\langle n-1 \mid 1\rangle\langle n \mid 1\rangle} A_{n-1}\left(2, \sigma_{2}, n-1, n\right) \times \\
& \sum_{\tilde{t}=2}^{n-2} \sum_{\sigma_{\tilde{t}}, \beta_{\tilde{t}} \in S_{n-4}} \mathcal{D}\left[2, \sigma_{2}, n-1, n \mid \widetilde{t}, \sigma_{\widetilde{t}}, n-1, n\right] \mathcal{S}\left[\sigma_{\widetilde{t}} \mid \beta_{\tilde{t}}\right]_{p_{n-1}} \\
& J_{\widetilde{t}, \dot{\alpha} \dot{\beta}}\left\{\mathcal{C}\left[\widetilde{t}, n-1, \beta_{\widetilde{t}}, n \mid 2, n-1, \beta_{2}, n\right] \widetilde{A}_{n-1}\left(2, n-1, \beta_{2}, n\right)\right\} \\
& =(-i) \sum_{\sigma_{2}, \beta_{2} \in S_{n-4}} \frac{\widetilde{\lambda}_{1}^{\dot{\alpha}} \widetilde{\lambda}_{1}^{\dot{\beta}}\langle n \mid n-1\rangle}{\langle n-1 \mid 1\rangle\langle n \mid 1\rangle} A_{n-1}\left(2, \sigma_{2}, n-1, n\right) \times \\
& \sum_{\tilde{t}=2}^{n-2} \sum_{\sigma_{\tilde{t}}, \beta_{\tilde{t}} \in S_{n-4}} \mathcal{D}\left[2, \sigma_{2}, n-1, n \mid \widetilde{t}, \sigma_{\widetilde{t}}, n-1, n\right] \mathcal{S}\left[\sigma_{\tilde{t}} \mid \beta_{\tilde{t}} p_{n-1}\right. \\
& \mathcal{C}\left[\widetilde{t}, n-1, \beta_{\widetilde{t}}, n \mid 2, n-1, \beta_{2}, n\right]\left\{\widetilde{J}_{\widetilde{t}, \dot{\alpha} \dot{\beta}} A_{n-1}\left(2, n-1, \beta_{2}, n\right)\right\} \\
& +(-i) \sum_{\sigma_{2}, \beta_{2} \in S_{n-4}} \frac{\widetilde{\lambda}_{1}^{\dot{\alpha}} \widetilde{\lambda}_{1}^{\dot{\beta}}\langle n \mid n-1\rangle}{\langle n-1 \mid 1\rangle\langle n \mid 1\rangle} A_{n-1}\left(2, \sigma_{2}, n-1, n\right) \times \\
& \sum_{\tilde{t}=2}^{n-2} \sum_{\sigma_{\tilde{t}}, \beta_{\tilde{t}} \in S_{n-4}} \mathcal{D}\left[2, \sigma_{2}, n-1, n \mid \widetilde{t}, \sigma_{\widetilde{t}}, n-1, n\right] \mathcal{S}\left[\sigma_{\widetilde{t}} \mid \beta_{\tilde{t}} p_{p_{n-1}}\right. \\
& \left\{J_{\widetilde{t}, \dot{\alpha} \dot{\beta}} \mathcal{C}\left[\widetilde{t}, n-1, \beta_{\widetilde{t}}, n \mid 2, n-1, \beta_{2}, n\right]\right\} \widetilde{A}_{n-1}\left(2, n-1, \beta_{2}, n\right) .
\end{aligned}
$$

For the first term in (4.14), if we have the following identity

$\sum_{\sigma_{\tilde{t}}, \beta_{\tilde{t}} \in S_{n-4}} \mathcal{D}\left[t, \sigma_{t}, n-1, n \mid \widetilde{t}, \sigma_{\tilde{t}}, n-1, n\right] \mathcal{S}\left[\sigma_{\tilde{t}} \mid \beta_{\tilde{t}}\right] p_{n-1} \mathcal{C}\left[\widetilde{t}, n-1, \beta_{\tilde{t}}, n \mid t, n-1, \beta_{t}, n\right]=\mathcal{S}\left[\sigma_{t} \mid \beta_{t}\right]_{p_{n-1}}$, 
the first term can be simplified as

$$
\begin{aligned}
& \sum_{\sigma_{2}, \beta_{2} \in S_{n-4}} \frac{\widetilde{\lambda}_{1}^{\dot{\alpha}} \widetilde{\lambda}_{1}^{\dot{\beta}}\langle n \mid n-1\rangle}{\langle n-1 \mid 1\rangle\langle n \mid 1\rangle} A_{n-1}\left(2, \sigma_{2}, n-1, n\right) \sum_{\widetilde{t}=2}^{n-2} \mathcal{S}\left[\sigma_{2} \mid \beta_{2}\right]_{p_{n-1}}\left\{J_{\widetilde{t}, \dot{\alpha} \dot{\beta}} \widetilde{A}_{n-1}\left(2, n-1, \beta_{2}, n\right)\right\} \\
= & \sum_{\sigma_{2}, \beta_{2} \in S_{n-4}} \frac{\widetilde{\lambda}_{1}^{\dot{\alpha}} \widetilde{\lambda}_{1}^{\dot{\beta}}\langle n \mid n-1\rangle}{\langle n-1 \mid 1\rangle\langle n \mid 1\rangle} A_{n-1}\left(2, \sigma_{2}, n-1, n\right) \mathcal{S}\left[\sigma_{2} \mid \beta_{2}\right]_{p_{n-1}}\left\{\left[\sum_{\tilde{t}=2}^{n-2} J_{\widetilde{t}, \dot{\alpha} \dot{\beta}}\right] \widetilde{A}_{n-1}\left(2, n-1, \beta_{2}, n\right)\right\} \\
= & 0,
\end{aligned}
$$

where we have used angular momentum conservation

$$
\begin{aligned}
& \widetilde{\lambda}_{1}^{\dot{\alpha}} \widetilde{\lambda}_{1}^{\dot{\beta}}\left\{\sum_{\widetilde{t}=2}^{n-2} J_{\widetilde{t}, \dot{\alpha} \dot{\beta}}\right\}\left\{\widetilde{A}_{n-1}\left(2, n-1, \beta_{2}, n\right)\right\}=\widetilde{\lambda}_{1}^{\dot{\alpha}} \widetilde{\lambda}_{1}^{\dot{\beta}}\left\{\sum_{\widetilde{t}=2}^{n} J_{\widetilde{t}, \dot{\alpha} \dot{\beta}}\right\}\left\{\widetilde{A}_{n-1}\left(2, n-1, \beta_{2}, n\right)\right\} \\
= & \left(-\widetilde{\lambda}_{1}^{\dot{\alpha}} \widetilde{\lambda}_{1}^{\dot{\beta}} J_{\widetilde{t}=1, \dot{\alpha} \dot{\beta}}\right)\left\{\widetilde{A}_{n-1}\left(2, n-1, \beta_{2}, n\right)\right\}=0 .
\end{aligned}
$$

For the second term in (4.14), if we have the following identity

$0=\sum_{\widetilde{t}=2}^{n-2} \sum_{\sigma_{\tilde{t}}, \beta_{\tilde{t}} \in S_{n-4}} \mathcal{D}\left[t, \sigma_{t}, n-1, n \mid \widetilde{t}, \sigma_{\widetilde{t}}, n-1, n\right] \mathcal{S}\left[\sigma_{\widetilde{t}} \mid \beta_{\widetilde{t}}\right]_{p_{n-1}} J_{\widetilde{t}, \dot{\alpha} \dot{\beta}}\left\{\mathcal{C}\left[\widetilde{t}, n-1, \beta_{\widetilde{t}}, n \mid t, n-1, \beta_{t}, n\right]\right\}$,

for arbitrary $t \in\{2,3, \ldots, n-2\}$ and related $\left\{\sigma_{t}, \beta_{t}\right\}$, the contribution vanishes also.

Identities (4.15) and (4.18) are the consistency requirement of the new soft graviton theorem and the old KLT formula. While the first identity can be understood from the changing of the basis (we will discuss it shortly), the second identity is very nontrivial. Currently, we do not have an analytic proof for them although in our few examples, we have checked them explicitly. We believe the knowledge of these two identities will tell us some important aspects of momentum kernel $\mathcal{S}[\alpha \mid \beta]$.

Now we present the physical understanding of the first identity (4.15). Noticing that we have many $(n-3)$ ! symmetry KLT forms. They are equivalent to each other, but it is hard to see that from the angle of BCJ relation for color-ordered Yang-Mills theory. In other words, we have

$$
\begin{aligned}
M_{n-1} & =\sum_{\sigma_{t}, \beta_{t} \in S_{n-4}} A_{n-1}\left(t, \sigma_{t}, n-1, n\right) \mathcal{S}\left[\sigma_{t} \mid \beta_{t}\right] p_{p_{n-1}} \widetilde{A}_{n-1}\left(t, n-1, \beta_{t}, n\right) \\
& =\sum_{\sigma_{\tilde{t}}, \beta_{\tilde{t}} \in S_{n-4}} A_{n-1}\left(\widetilde{t}, \sigma_{\widetilde{t}}, n-1, n\right) \mathcal{S}\left[\sigma_{\widetilde{t}} \mid \beta_{\widetilde{t}}\right] p_{n-1} \widetilde{A}_{n-1}\left(\widetilde{t}, n-1, \beta_{\widetilde{t}}, n\right)
\end{aligned}
$$

where $\sigma_{t}, \beta_{t}$ is the set of removing element $t$ from $\{2,3, \ldots, n-2\}$. Plugging the transformation of basis (4.13) back, we have

$$
\begin{aligned}
& \sum_{\sigma_{\tilde{t}}, \beta_{\tilde{t}} \in S_{n-4}} A_{n-1}\left(\widetilde{t}, \sigma_{\widetilde{t}}, n-1, n\right) \mathcal{S}\left[\sigma_{\widetilde{t}} \mid \beta_{\tilde{t}}\right] p_{n-1} \widetilde{A}_{n-1}\left(\widetilde{t}, n-1, \beta_{\widetilde{t}}, n\right) \\
= & \sum_{\sigma_{\tilde{t}}, \beta_{\tilde{t}} \in S_{n-4}}\left\{\sum_{\sigma_{t} \in S_{n-4}} A_{n-1}\left(t, \sigma_{t}, n-1, n\right) \mathcal{D}\left[t, \sigma_{t}, n-1, n \mid \widetilde{t}, \sigma_{\widetilde{t}}, n-1, n\right]\right\} \mathcal{S}\left[\sigma_{\tilde{t}} \mid \beta_{\tilde{t}}\right] p_{n-1}
\end{aligned}
$$




$$
\begin{aligned}
& \left\{\sum_{\beta_{t} \in S_{n-4}} \mathcal{C}\left[\widetilde{t}, n-1, \beta_{\widetilde{t}}, n \mid t, n-1, \beta_{t}, n\right] \widetilde{A}_{n-1}\left(t, n-1, \beta_{t}, n\right)\right\} \\
= & \sum_{\sigma_{t} \in S_{n-4}} A_{n-1}\left(t, \sigma_{t}, n-1, n\right)\left\{\sum_{\sigma_{\tilde{t}}, \beta_{\tilde{t}} \in S_{n-4}} \mathcal{D}\left[t, \sigma_{t}, n-1, n \mid \widetilde{t}, \sigma_{\widetilde{t}}, n-1, n\right] \mathcal{S}\left[\sigma_{\widetilde{t}} \mid \beta_{\tilde{t}}\right] p_{n-1} \times\right. \\
\left.\mathcal{C}\left[\widetilde{t}, n-1, \beta_{\widetilde{t}}, n \mid t, n-1, \beta_{t}, n\right]\right\} \times & \\
& \widetilde{A}_{n-1}\left(t, n-1, \beta_{t}, n\right)
\end{aligned}
$$

Because the independence of the BCJ basis, we should obtain the identity (4.15).

\subsection{The sub-sub-leading part from KLT relation}

Now we consider the sub-sub-leading order. From the KLT formula, we have

$$
\begin{aligned}
& \left(\epsilon^{-2} A_{L, 0}+\epsilon^{-1} A_{L, 1}+\epsilon^{0} A_{L, 2}+\ldots\right)\left(\epsilon \mathcal{S}_{0}+\epsilon^{2} \mathcal{S}_{1}+\epsilon^{3} \mathcal{S}_{2}+\ldots\right)\left(\epsilon^{-2} A_{R, 0}+\epsilon^{-1} A_{R, 1}+\epsilon^{0} A_{R, 2}+\ldots\right) \\
= & \epsilon^{-3} A_{L, 0} \mathcal{S}_{0} A_{R, 0}+\epsilon^{-2}\left(A_{L, 1} \mathcal{S}_{0} A_{R, 0}+A_{L, 0} \mathcal{S}_{1} A_{R, 0}+A_{L, 0} \mathcal{S}_{0} A_{R, 1}\right) \\
& +\epsilon^{-1}\left(A_{L, 2} \mathcal{S}_{0} A_{R, 0}+A_{L, 0} \mathcal{S}_{2} A_{R, 0}+A_{L, 0} \mathcal{S}_{0} A_{R, 2}+A_{L, 1} \mathcal{S}_{1} A_{R, 0}+A_{L, 0} \mathcal{S}_{1} A_{R, 1}+A_{L, 1} \mathcal{S}_{0} A_{R, 1}\right)+\ldots
\end{aligned}
$$

Thus we see that to use this formula to study the sub-sub-leading singularity, we need to get the information of $\epsilon^{0} A_{L, 2}$, which does not have the universal structure and has not been fully discussed.

\section{Examples}

Having the general frame in previous section, we will present a few examples to demonstrate our ideas. In this section, we will give examples of $n=5,6$ while the more complicated example of $n=7$ will be given in the appendix.

\subsection{The case $n=5$}

Following our convention, in the stripped amplitude, $\tilde{\lambda}_{4}$ and $\tilde{\lambda}_{5}$ should be replaced by

$$
\tilde{\lambda}_{4}=-\sum_{k=2,3} \frac{\langle 5 \mid k\rangle}{\langle 5 \mid 4\rangle} \tilde{\lambda}_{k}-\epsilon \frac{\langle 5 \mid 1\rangle}{\langle 5 \mid 4\rangle} \tilde{\lambda}_{1}, \tilde{\lambda}_{5}=-\sum_{k=2,3} \frac{\langle 4 \mid k\rangle}{\langle 4 \mid 5\rangle} \tilde{\lambda}_{k}-\epsilon \frac{\langle 4 \mid 1\rangle}{\langle 4 \mid 5\rangle} \tilde{\lambda}_{1} .
$$

In particular that $\frac{d}{d \tilde{\lambda}_{4}^{\dot{\beta}}} \tilde{A}=\frac{d}{d \tilde{\lambda}_{5}^{\dot{\beta}}} \tilde{A}=0$, and therefore $J_{4 \dot{\alpha} \dot{\beta}} \tilde{A}=J_{5 \dot{\alpha} \dot{\beta}} \tilde{A}=0$. At 5-points it is relatively straightforward to write down all of the terms in $\Delta$ as

$$
\begin{aligned}
\Delta_{n=5}= & \frac{\widetilde{\lambda}_{1}^{\dot{\alpha}} \widetilde{\lambda}_{1}^{\dot{\beta}}\langle 5 \mid 4\rangle}{\langle 4 \mid 1\rangle\langle 5 \mid 1\rangle}\left\{A_{4}(2,3,4,5) \mathcal{S}[3 \mid 3]_{p_{4}}\left(J_{2, \dot{\alpha} \dot{\beta}} \widetilde{A}_{4}(2,4,3,5)\right)\right. \\
& \left.+A_{4}(3,2,4,5) \mathcal{S}[2 \mid 2]_{p_{4}}\left(J_{3, \dot{\alpha} \dot{\beta}} \widetilde{A}_{4}(3,4,2,5)\right)\right\} \\
= & \frac{\widetilde{\lambda}_{1}^{\dot{\alpha}} \widetilde{\lambda}_{1}^{\dot{\beta}}\langle 5 \mid 4\rangle}{\langle 4 \mid 1\rangle\langle 5 \mid 1\rangle}\left\{A_{4}(2,3,4,5) s_{34}\left(J_{2, \dot{\alpha} \dot{\beta}} \widetilde{A}_{4}(2,4,3,5)\right)+A_{4}(3,2,4,5) s_{24}\left(J_{3, \dot{\alpha} \dot{\beta}} \widetilde{A}_{4}(3,4,2,5)\right)\right\}
\end{aligned}
$$


For simplicity, we suppress overall factors $(-i)$ from the $\Delta$ here and in the following discussions. Now we do the changing of basis, i.e., using the BCJ relation to write

$$
\begin{aligned}
& A_{4}(3,2,4,5)=\frac{s_{34}}{s_{24}} A_{4}(2,3,4,5) \\
& \tilde{A}_{4}(3,4,2,5)=(-)^{4} \tilde{A}_{4}(5,2,4,3)=\tilde{A}_{4}(2,4,3,5)
\end{aligned}
$$

Plugging them back we get

$$
\Delta_{n=5}=\frac{\widetilde{\lambda}_{1}^{\dot{\alpha}} \widetilde{\lambda}_{1}^{\dot{\beta}}\langle 5 \mid 4\rangle}{\langle 4 \mid 1\rangle\langle 5 \mid 1\rangle} A_{4}(3,2,4,5) s_{2 \widehat{4}}\left\{\left(J_{3, \dot{\alpha} \dot{\beta}}+J_{2, \dot{\alpha} \dot{\beta}}\right) \widetilde{A}_{4}(3,4,2,5)\right\}=0
$$

by angular momentum conservation $\sum_{i=2}^{5} J_{i} \widetilde{A}=0$ (where $J_{4} \widetilde{A}=J_{5} \widetilde{A}=0$ has been used). For this case, two identities (4.15) and (4.18) are trivial to check.

\subsection{The case $n=6$}

For $n=6$ the difference term $\Delta_{n=6}$ splits into three parts: $t=2,3$ and 4 ,

$$
\Delta_{n=6}=\Delta_{n=6}^{t=2}+\Delta_{n=6}^{t=3}+\Delta_{n=6}^{t=4}
$$

where we have solved

$$
\tilde{\lambda}_{5}=-\sum_{k=2,3,4} \frac{\langle 6 \mid k\rangle}{\langle 6 \mid 5\rangle} \tilde{\lambda}_{k}-\epsilon \frac{\langle 6 \mid 1\rangle}{\langle 6 \mid 5\rangle} \tilde{\lambda}_{1}, \quad \tilde{\lambda}_{6}=-\sum_{k=2,3,4} \frac{\langle 5 \mid k\rangle}{\langle 5 \mid 6\rangle} \tilde{\lambda}_{k}-\epsilon \frac{\langle 5 \mid 1\rangle}{\langle 5 \mid 6\rangle} \tilde{\lambda}_{1}
$$

For simplicity in the following discussion we further suppress a common factor $(-)^{n+1} \frac{\langle n \mid n-1\rangle}{\langle n-1 \mid 1\rangle\langle n \mid 1\rangle} \tilde{\lambda}_{1}^{\alpha} \tilde{\lambda}_{1}^{\dot{\beta}}$ from the difference terms, thus we can write

$$
\begin{aligned}
\Delta_{n=6}^{t=2}= & A_{5}(2,3,4,5,6) \mathcal{S}[3,4 \mid 3,4]_{p_{5}}\left(J_{2} \widetilde{A}_{5}(2,5,3,4,6)\right) \\
& +A_{5}(2,3,4,5,6) \mathcal{S}[3,4 \mid 4,3]_{p_{5}}\left(J_{2} \widetilde{A}_{5}(2,5,4,3,6)\right) \\
& +A_{5}(2,4,3,5,6) \mathcal{S}[4,3 \mid 3,4]_{p_{5}}\left(J_{2} \widetilde{A}_{5}(2,5,3,4,6)\right) \\
& +A_{5}(2,4,3,5,6) \mathcal{S}[4,3 \mid 4,3]_{p_{5}}\left(J_{2} \widetilde{A}_{5}(2,5,4,3,6)\right) \\
\Delta_{n=6}^{t=3}= & A_{5}(3,2,4,5,6) \mathcal{S}[2,4 \mid 2,4]_{p_{5}}\left(J_{3} \widetilde{A}_{5}(3,5,2,4,6)\right) \\
& +A_{5}(3,2,4,5,6) \mathcal{S}[2,4 \mid 4,2]_{p_{5}}\left(J_{3} \widetilde{A}_{5}(3,5,4,2,6)\right) \\
& +A_{5}(3,4,2,5,6) \mathcal{S}[4,2 \mid 2,4]_{p_{5}}\left(J_{3} \widetilde{A}_{5}(3,5,2,4,6)\right) \\
& +A_{5}(3,4,2,5,6) \mathcal{S}[4,2 \mid 4,2]_{p_{5}}\left(J_{3} \widetilde{A}_{5}(3,5,4,2,6)\right) \\
\Delta_{n=6}^{t=4}= & A_{5}(4,2,3,5,6) \mathcal{S}[2,3 \mid 2,3]_{p_{5}}\left(J_{4} \widetilde{A}_{5}(4,5,2,3,6)\right) \\
& +A_{5}(4,2,3,5,6) \mathcal{S}[2,3 \mid 3,2]_{p_{5}}\left(J_{4} \widetilde{A}_{5}(4,5,3,2,6)\right) \\
& +A_{5}(4,3,2,5,6) \mathcal{S}[3,2 \mid 2,3]_{p_{5}}\left(J_{4} \widetilde{A}_{5}(4,5,2,3,6)\right) \\
& +A_{5}(4,3,2,5,6) \mathcal{S}[3,2 \mid 3,2]_{p_{5}}\left(J_{4} \widetilde{A}_{5}(4,5,3,2,6)\right)
\end{aligned}
$$

Now we translate all amplitudes $A$ into the basis $\{A(6,2,4,3,5), A(6,2,3,4,5)\}$

$$
A_{5}(6,4,2,3,5)=\frac{\left(s_{43}+s_{45}\right) A_{5}(6,2,4,3,5)+s_{45} A_{5}(6,2,3,4,5)}{s_{46}}
$$




$$
\begin{aligned}
& A_{5}(6,3,2,4,5)=\frac{\left(s_{34}+s_{35}\right) A_{5}(6,2,3,4,5)+s_{35} A_{5}(6,2,4,3,5)}{s_{36}} \\
& A_{5}(6,4,3,2,5)=\frac{-s_{24} s_{35} A_{5}(6,2,4,3,5)-s_{45}\left(s_{25}+s_{23}\right) A_{5}(6,2,3,4,5)}{s_{46} s_{25}} \\
& A_{5}(6,3,4,2,5)=\frac{-s_{23} s_{45} A_{5}(6,2,3,4,5)-s_{35}\left(s_{25}+s_{24}\right) A_{5}(6,2,4,3,5)}{s_{36} s_{25}}
\end{aligned}
$$

and all amplitudes $\widetilde{A}_{5}$ into the basis $\left\{\widetilde{A}_{5}(2,5,3,4,6), \widetilde{A}_{5}(2,5,4,3,6)\right\}$

$$
\begin{gathered}
\widetilde{A}_{5}(3,5,2,4,6)=\frac{-\widetilde{A}_{5}(2,5,3,4,6)\left(s_{45}+s_{43}\right)-\widetilde{A}_{5}(2,5,4,3,6) s_{45}}{s_{24}} \\
\widetilde{A}_{5}(4,5,2,3,6)=\frac{-\widetilde{A}_{5}(2,5,4,3,6)\left(s_{35}+s_{43}\right)-\widetilde{A}_{5}(2,5,3,4,6) s_{35}}{s_{23}} \\
\left.\widetilde{A}_{5}(3,5,4,2,6)\right)=\frac{-\left(s_{43}+s_{46}\right) \widetilde{A}_{5}(2,5,4,3,6)-s_{46} \widetilde{A}_{5}(2,5,3,4,6)}{s_{24}} \\
\left.\widetilde{A}_{5}(4,5,3,2,6)\right)=\frac{-\left(s_{43}+s_{36}\right) \widetilde{A}_{5}(2,5,3,4,6)-s_{36} \widetilde{A}_{5}(2,5,4,3,6)}{s_{23}}
\end{gathered}
$$

Putting it back with some calculation we have

$$
\begin{aligned}
\Delta_{n=6}^{t=3}= & A_{5}(2,3,4,5,6)\left\{-s_{45}\left(s_{23}+s_{25}\right)\left(J_{3, \dot{\alpha} \dot{\beta}} \frac{-\widetilde{A}_{5}(2,5,3,4,6)\left(s_{45}+s_{43}\right)-\widetilde{A}_{5}(2,5,4,3,6) s_{45}}{s_{24}}\right)\right. \\
& \left.+s_{45} s_{26}\left(J_{3, \dot{\alpha} \dot{\beta}} \frac{-\left(s_{43}+s_{46}\right) \widetilde{A}_{5}(2,5,4,3,6)-s_{46} \widetilde{A}_{5}(2,5,3,4,6)}{s_{24}}\right)\right\} \\
& +A_{5}(2,4,3,5,6)\left\{-s_{35} s_{24}\left(J_{3, \dot{\alpha} \dot{\beta}} \frac{-\widetilde{A}_{5}(2,5,3,4,6)\left(s_{45}+s_{43}\right)-\widetilde{A}_{5}(2,5,4,3,6) s_{45}}{s_{24}}\right)\right\}
\end{aligned}
$$

Further simplification by using ( notice that $J_{3, \dot{\alpha} \dot{\beta}} s_{24}=0$ )

$$
\begin{aligned}
& \left(s_{23}+s_{25}\right)\left(J_{3, \dot{\alpha} \dot{\beta}}\left(s_{45}+s_{43}\right)\right)-s_{26}\left(J_{3, \dot{\alpha} \dot{\beta}} s_{46}\right)=s_{24}\left(J_{3, \dot{\alpha} \dot{\beta}} s_{46}\right) \\
& \left(s_{23}+s_{25}\right)\left(J_{3, \dot{\alpha} \dot{\beta}} s_{45}\right)-s_{26}\left(J_{3, \dot{\alpha} \dot{\beta}}\left(s_{43}+s_{46}\right)\right)=-s_{24}\left(J_{3, \dot{\alpha} \dot{\beta}} s_{45}\right)
\end{aligned}
$$

leads

$$
\begin{aligned}
& \Delta_{n=6}^{t=3}=A_{5}(2,3,4,5,6)\left\{\mathcal{S}[3,4 \mid 3,4]_{p_{5}}\left(J_{3, \dot{\alpha} \dot{\beta}} \widetilde{A}_{5}(2,5,3,4,6)\right)+\mathcal{S}[3,4 \mid 4,3]_{p_{5}}\left(J_{3, \dot{\alpha} \dot{\beta}} \widetilde{A}_{5}(2,5,4,3,6)\right)\right. \\
& \left.+s_{45}\left(J_{3, \dot{\alpha} \dot{\beta}} s_{46}\right) \widetilde{A}_{5}(2,5,3,4,6)-s_{45} \widetilde{A}_{5}(2,5,4,3,6)\left(J_{3, \dot{\alpha} \dot{\beta}} s_{45}\right)\right\} \\
& +A_{5}(2,4,3,5,6)\left\{\mathcal{S}[4,3 \mid 3,4]_{p_{5}}\left(J_{3, \dot{\alpha} \dot{\beta}} \widetilde{A}_{5}(2,5,3,4,6)\right)+\mathcal{S}[4,3 \mid 4,3]_{p_{5}}\left(J_{3, \dot{\alpha} \dot{\beta}} \widetilde{A}_{5}(2,5,4,3,6)\right)\right. \\
& \left.-s_{35} \widetilde{A}_{5}(2,5,3,4,6)\left(J_{3, \dot{\alpha} \dot{\beta}} s_{46}\right)+s_{35} \widetilde{A}_{5}(2,5,4,3,6)\left(J_{3, \dot{\alpha} \dot{\beta}} s_{45}\right)\right\}
\end{aligned}
$$

Notice that part of them (i.e., the part with $J$ acting only on $\widetilde{A}$ ) is exactly the same as $\Delta_{n=6}^{t=2}$ except the $J_{2, \dot{\alpha} \dot{\beta}}$ is replaced by $J_{3, \dot{\alpha} \dot{\beta}}$. It is nothing, but the explicit checking the identity (4.15) with $t=2, \widetilde{t}=3$.

Doing similar calculation we found

$$
\Delta_{n=6}^{t=4}=A_{5}(2,3,4,5,6)\left\{\mathcal{S}[3,4 \mid 3,4]_{p_{5}}\left(J_{4, \dot{\alpha} \dot{\beta}} \widetilde{A}_{5}(2,5,3,4,6)\right)+\mathcal{S}[3,4 \mid 4,3]_{p_{5}}\left(J_{4, \dot{\alpha} \dot{\beta}} \widetilde{A}_{5}(2,5,4,3,6)\right)\right.
$$




$$
\begin{aligned}
& \left.+s_{45}\left(J_{4, \dot{\alpha} \dot{\beta}} s_{35}\right) \widetilde{A}_{5}(2,5,3,4,6)+s_{45} \widetilde{A}_{5}(2,5,4,3,6)\left(J_{4, \dot{\alpha} \dot{\beta}}\left(s_{34}+s_{35}\right)\right)\right\} \\
& +A_{5}(2,4,3,5,6)\left\{\mathcal{S}[4,3 \mid 3,4]_{p_{5}}\left(J_{4, \dot{\alpha} \dot{\beta}} \widetilde{A}_{5}(2,5,3,4,6)\right)+\mathcal{S}[4,3 \mid 4,3]_{p_{5}}\left(J_{4, \dot{\alpha} \dot{\beta}} \widetilde{A}_{5}(2,5,4,3,6)\right)\right. \\
& \left.-s_{35} \widetilde{A}_{5}(2,5,3,4,6)\left(J_{4, \dot{\alpha} \dot{\beta}} s_{35}\right)+s_{35} \widetilde{A}_{5}(2,5,4,3,6)\left(J_{4, \dot{\alpha} \dot{\beta}} s_{36}\right)\right\}
\end{aligned}
$$

where again the identity (4.15) with $t=2, \widetilde{t}=4$ has been checked. Thus when we sum up three terms $\Delta_{n=6}^{t=2}, \Delta_{n=6}^{t=3}, \Delta_{n=6}^{t=4}$, the part with $J$ acting directly on $\widetilde{A}$ vanishes by angular momentum conservation and we are left with

$$
\begin{aligned}
R= & A_{5}(2,3,4,5,6)\left\{+s_{45}\left(J_{3, \dot{\alpha} \dot{\beta}} s_{46}\right) \widetilde{A}_{5}(2,5,3,4,6)-s_{45} \widetilde{A}_{5}(2,5,4,3,6)\left(J_{3, \dot{\alpha} \dot{\beta}} s_{45}\right)\right. \\
& \left.+s_{45}\left(J_{4, \dot{\alpha} \dot{\beta}} s_{35}\right) \widetilde{A}_{5}(2,5,3,4,6)-s_{45} \widetilde{A}_{5}(2,5,4,3,6)\left(J_{4, \dot{\alpha} \dot{\beta}} s_{36}\right)\right\} \\
& +A_{5}(2,4,3,5,6)\left\{-s_{35} \widetilde{A}_{5}(2,5,3,4,6)\left(J_{3, \dot{\alpha} \dot{\beta}} s_{46}\right)+s_{35} \widetilde{A}_{5}(2,5,4,3,6)\left(J_{3, \dot{\alpha} \dot{\beta}} s_{45}\right)\right. \\
& \left.-s_{35} \widetilde{A}_{5}(2,5,3,4,6)\left(J_{4, \dot{\alpha} \dot{\beta}} s_{35}\right)+s_{35} \widetilde{A}_{5}(2,5,4,3,6)\left(J_{4, \dot{\alpha} \dot{\beta}} s_{36}\right)\right\}
\end{aligned}
$$

where $J$ acts only on $s_{i j}$. Using

$$
\begin{aligned}
& \left.J_{3, \dot{\alpha} \dot{\beta}} s_{i 5}=\frac{-i}{2}\langle 5 \mid i\rangle \frac{\langle 6 \mid 3\rangle}{\langle 6 \mid 5\rangle} \widetilde{\lambda}_{3,(\dot{\alpha}} \widetilde{\lambda}_{i, \dot{\beta})}, J_{3, \dot{\alpha} \dot{\beta}} s_{i 6}=\frac{-i}{2}\langle 6 \mid i\rangle \frac{\langle 5 \mid 3\rangle}{\langle 5 \mid 6\rangle} \widetilde{\lambda}_{3,(\dot{\alpha}} \widetilde{\lambda}_{i, \dot{\beta}}\right) \\
& J_{3, \dot{\alpha} \dot{\beta}} s_{i 3}=+\frac{i}{2}\langle 3 \mid i\rangle \widetilde{\lambda}_{3,(\dot{\alpha}} \widetilde{\lambda}_{i, \dot{\beta})}, i=2,4 \\
& J_{4, \dot{\alpha} \dot{\beta}} s_{i 5}=\frac{-i}{2}\langle 5 \mid i\rangle \frac{\langle 6 \mid 4\rangle}{\langle 6 \mid 5\rangle} \widetilde{\lambda}_{4,(\dot{\alpha}} \widetilde{\lambda}_{i, \dot{\beta})}, J_{4, \dot{\alpha} \dot{\beta}} s_{i 6}=\frac{-i}{2}\langle 6 \mid i\rangle \frac{\left.\langle 5 \mid 4\rangle \widetilde{\lambda}_{4,(\dot{\alpha}} \widetilde{\lambda}_{i, \dot{\beta}}\right)}{\langle 5 \mid 6\rangle}, \\
& J_{4, \dot{\alpha} \dot{\beta}} s_{i 4}=+\frac{i}{2}\langle 4 \mid i\rangle \widetilde{\lambda}_{4,(\dot{\alpha}} \widetilde{\lambda}_{i, \dot{\beta})}, i=2,3
\end{aligned}
$$

we see immediately that $R=0$. In other words, we have explicitly checked the second identity (4.18) for the special case.

\section{Conclusion}

In this paper, we studied the new soft graviton theorem from the angle of KLT relation. We have demonstrated that how the new soft gluon theorem are glued together by KLT formula to produce the corresponding soft theorem for gravity. In the process, two important identities (4.15) and (4.18) has been observed.

There are a lot of open questions deserve to be investigated. First, the two identities need an analytic proof. Secondly, the sub-sub-leading soft factor in KLT relation should be understood. Although at this order, contributions from non-universal soft part of color ordered Yang-Mills amplitudes appear, we guess that their effects will be canceled out by nice property of momentum kernel $\mathcal{S}$. It will be fascinating to see how it happens. Thirdly, in this paper, we have focused on the $4 \mathrm{D}$, it will be interesting to discuss it in general dimension since KLT formula holds in general dimension. Finally, there are also other general formulas for gravity amplitudes (such as these given in [40-42] ) and it will be nice to see how the new soft graviton theorem makes its appearance. 


\section{Acknowledgements}

Y. J. Du would like to acknowledge the EU programme Erasmus Mundus Action 2, Project 9 and the International Postdoctoral Exchange Fellowship Program of China for supporting his postdoctoral research in Lund University (with Fudan University as the home university). Y. J. Du's research is supported in parts by the NSF of China Grant No.11105118, China Postdoctoral Science Foundation No.2013M530175 and the Fundamental Research Funds for the Central Universities of Fudan University No.20520133169. C. F. is grateful for Y-T Huang for the helpful discussions. The work of C. F. was supported from National Science Council, 50 billions project of Ministry of Education and National Center for Theoretical Science, Taiwan, Republic of China as well as the support from S.T. Yau center of National Chiao Tung University. Y.W. would like acknowledge the support from NSF grant PHY-1316617. B.F is supported, in part, by fund from Qiu-Shi and Chinese NSF funding under contract No.11031005, No.11135006, No. 11125523.

\section{A Example with $n=7$}

In this appendix we verify that identities (4.15) and (4.18) are holding at $n=7$. Our strategy used in previous examples applies to 7-points, although the complexity involved increases considerably. As in the previous examples, we choose to work in a convenient minimum basis $\tilde{A}\left(2,6, \beta_{2}, 7\right), A\left(2, \sigma_{2}, 6,7\right)$ (Here we use $A$ instead of $A_{6}$ for short), i.e., do the following transformation with $t=3,4,5$ :

$$
\begin{aligned}
\tilde{A}\left(t, 6, \beta_{t}, 7\right) & =\sum_{\beta_{t} \in S_{3}} \mathcal{C}\left[t, 6, \beta_{t}, 7 \mid 2,6, \beta_{2}, 7\right] \tilde{A}\left(2,6, \beta_{2}, 7\right), \\
A\left(t, \sigma_{t}, 6,7\right) & =\sum_{\sigma_{t} \in S_{3}} A\left(2, \sigma_{2}, 6,7\right) \mathcal{D}\left[2, \sigma_{2}, 6,7 \mid t, \sigma, 6,7\right]
\end{aligned}
$$

Our task then amounts to showing that, for both identities, terms associated with each independent product of basis amplitudes $A \tilde{A}$ match accordingly for both sides of the equations (4.15) and (4.18). In the discussion below we focus on terms containing $\tilde{A}(2,6,3,4,5,7)$, namely when $\beta_{2}=\{3,4,5\}$. The rest of the coefficients follow similar argument up to permutations of $\{3,4,5\}$. In principle it is straightforward to work out all translation coefficients $\mathcal{C}, \mathcal{D}$ and check if the identities are holding. However we can perform the calculation in a slightly more organized manner. In particular note that common factors are quite often shared between different translation coefficients.

For the purpose of demonstration let us consider translating a specific amplitude $\tilde{A}(3,6,2,4,5,7)$ into minimum basis. This can be done by first expressing the amplitude in the $\tilde{A}(2, \ldots, 7)$ Kleiss-Klein (KK) basis that fixes legs 2 and 7 at both ends, and then subsequently translating to the $\tilde{A}(2,6, \ldots, 7)$ minimum basis of interest where legs 6 and 2 are adjacent:

$$
\begin{aligned}
\tilde{A}(3,6,2,4,5,7)= & \tilde{A}(2,4,5,6,3,7)+\tilde{A}(2,4,6,5,3,7)+\tilde{A}(2,4,6,3,5,7)+\tilde{A}(2,6,4,5,3,7) \\
& +\tilde{A}(2,6,4,3,5,7)+\tilde{A}(2,6,3,4,5,7)
\end{aligned}
$$




$$
\begin{aligned}
= & \left(1-\frac{\left(s_{42}+s_{46}+s_{43}\right)}{s_{42}}+E[45,3 \mid 345]\right) \tilde{A}(2,6,3,4,5,7) \\
& +\ldots(\text { terms not contributing to } \tilde{A}(2,6,3,4,5,7)),
\end{aligned}
$$

where in the third line we used BCJ relation to remove the ill-favored leg 4 between 2 and 6 in the next to adjacent amplitude $\tilde{A}(2,4,6,5,3,7)$, and we introduced the shorthand notation $E[45,3 \mid 345]$ to denote the next-to-next-to adjacent expansion coefficient,

$$
\tilde{A}(2,\{4,5\}, 6,\{3\}, 7)=\sum_{\sigma} E[45,3 \mid \sigma] \tilde{A}(2,6, \sigma, 7) .
$$

The coefficient $E[45,3 \mid 345]$ can be determined from simultaneous equations consisting of BCJ relations, yielding

$$
\begin{gathered}
E[45,3 \mid 345]=\frac{(-1)}{s_{42} s_{52}-\left(s_{42}+s_{45}\right)\left(s_{52}+s_{54}\right)}\left[-\left(s_{42}+s_{45}+s_{46}+s_{43}\right)\left(s_{52}+s_{56}+s_{53}+s_{54}\right)\right. \\
\left.+\frac{\left(s_{42}+s_{45}\right)\left(s_{52}+s_{54}+s_{56}+s_{53}\right)\left(s_{42}+s_{46}+s_{43}\right)}{s_{42}}\right]
\end{gathered}
$$

All translation coefficients can be determined via similar procedures. Explicitly we have, for the $t=3$ sector,

$$
\begin{aligned}
& \mathcal{C}[3,6,2,4,5,7 \mid 2,6,3,4,5,7]=1-\frac{\left(s_{42}+s_{46}+s_{43}\right)}{s_{42}}+E[45,3] \\
& \mathcal{C}[3,6,2,5,4,7 \mid 2,6,3,4,5,7]=-\frac{\left(s_{52}+s_{56}+s_{53}+s_{54}\right)}{s_{52}}+E[54,3] \\
& \mathcal{C}[3,6,4,2,5,7 \mid 2,6,3,4,5,7]=\frac{\left(s_{42}+s_{46}+s_{43}\right)}{s_{42}}-E[45,3]-E[54,3] \\
& \mathcal{C}[3,6,4,5,2,7 \mid 2,6,3,4,5,7]=E[54,3] \\
& \mathcal{C}[3,6,5,2,4,7 \mid 2,6,3,4,5,7]=\frac{\left(s_{52}+s_{56}+s_{53}+s_{54}\right)}{s_{52}}-E[45,3]-E[54,3] \\
& \mathcal{C}[3,6,5,4,2,7 \mid 2,6,3,4,5,7]=E[45,3] .
\end{aligned}
$$

For $t=4$ we have

$$
\begin{aligned}
& \mathcal{C}[4,6,2,3,5,7 \mid 2,6,3,4,5,7]=1-\frac{\left(s_{32}+s_{36}\right)}{s_{32}}+E[35,4] \\
& \mathcal{C}[4,6,2,5,3,7 \mid 2,6,3,4,5,7]=-\frac{\left(s_{52}+s_{56}+s_{53}+s_{54}\right)}{s_{52}}+E[53,4] \\
& \mathcal{C}[4,6,3,2,5,7 \mid 2,6,3,4,5,7]=\frac{\left(s_{32}+s_{36}\right)}{s_{32}}-E[35,4]-E[53,4] \\
& \mathcal{C}[4,6,3,5,2,7 \mid 2,6,3,4,5,7]=E[53,4] \\
& \mathcal{C}[4,6,5,2,3,7 \mid 2,6,3,4,5,7]=\frac{\left(s_{52}+s_{56}+s_{53}+s_{54}\right)}{s_{52}}-E[35,4]-E[53,4] \\
& \mathcal{C}[4,6,5,3,2,7 \mid 2,6,3,4,5,7]=E[35,4]
\end{aligned}
$$

and similarly for $t=5$,

$$
\mathcal{C}[5,6,2,3,4,7 \mid 2,6,3,4,5,7]=1-\frac{\left(s_{32}+s_{36}\right)}{s_{32}}+E[34,5]
$$




$$
\begin{aligned}
& \mathcal{C}[5,6,2,4,3,7 \mid 2,6,3,4,5,7]=-\frac{\left(s_{42}+s_{46}+s_{43}\right)}{s_{42}}+E[43,5] \\
& \mathcal{C}[5,6,3,2,4,7 \mid 2,6,3,4,5,7]=\frac{\left(s_{32}+s_{36}\right)}{s_{32}}-E[34,5]-E[43,5] \\
& \mathcal{C}[5,6,3,4,2,7 \mid 2,6,3,4,5,7]=E[43,5] \\
& \mathcal{C}[5,6,4,2,3,7 \mid 2,6,3,4,5,7]=\frac{\left(s_{42}+s_{46}+s_{43}\right)}{s_{42}}-E[34,5]-E[43,5] \\
& \mathcal{C}[5,6,4,3,2,7 \mid 2,6,3,4,5,7]=E[34,5],
\end{aligned}
$$

whereas the next-to-next-to adjacent expansion coefficients are given by

$$
\begin{aligned}
E[54,3]= & \frac{(-1)}{s_{52} s_{42}-\left(s_{52}+s_{54}\right)\left(s_{42}+s_{45}\right)}\left[-\left(s_{52}+s_{54}+s_{56}+s_{53}\right)\left(s_{42}+s_{46}+s_{43}\right)\right. \\
& +\frac{\left(s_{52}+s_{54}\right)\left(s_{42}+s_{45}+s_{46}+s_{43}\right)\left(s_{52}+s_{56}+s_{53}+s_{54}\right)}{s_{52}}\left[-\left(s_{32}+s_{35}+s_{36}\right)\left(s_{52}+s_{56}+s_{53}+s_{54}\right)\right. \\
E[35,4]= & \left.\frac{(-1)}{s_{32} s_{52}-\left(s_{32}+s_{35}\right)\left(s_{52}+s_{53}\right)}\right] \\
& +\frac{\left(s_{32}+s_{35}\right)\left(s_{52}+s_{53}+s_{56}+s_{54}\right)\left(s_{32}+s_{36}\right)}{s_{32}}\left[-\left(s_{52}+s_{53}+s_{56}+s_{54}\right)\left(s_{32}+s_{36}\right)\right. \\
E[53,4]= & \frac{(\mathrm{A} .1)}{s_{52} s_{32}-\left(s_{52}+s_{53}\right)\left(s_{32}+s_{35}\right)}\left[-\left(s_{32}+s_{34}+s_{36}\right)\left(s_{42}+s_{46}+s_{43}\right)\right. \\
& \left.+\frac{\left(s_{52}+s_{53}\right)\left(s_{32}+s_{35}+s_{36}\right)\left(s_{52}+s_{56}+s_{53}+s_{54}\right)}{s_{52}}\right] \\
E[34,5]= & \frac{(\mathrm{A} .16)}{s_{32} s_{42}-\left(s_{32}+s_{34}\right)\left(s_{42}+s_{43}\right)}\left[s_{32}+s_{36}\right) \\
& \left.+\frac{\left(s_{32}+s_{34}\right)\left(s_{42}+s_{43}+s_{46}\right)\left(s_{32}\right.}{s_{32}}\right] \\
E[43,5]= & \frac{(-1)}{s_{42} s_{32}-\left(s_{42}+s_{43}\right)\left(s_{32}+s_{34}\right)}\left[-\left(s_{42}+s_{43}+s_{46}\right)\left(s_{32}+s_{36}\right)\right. \\
& \left.+\frac{\left(s_{42}+s_{43}\right)\left(s_{32}+s_{34}+s_{36}\right)\left(s_{42}+s_{46}+s_{43}\right)}{s_{42}}\right]
\end{aligned}
$$

Identity (4.15). Let us first verify identity (4.15) for the case when $\beta_{2}=\{345\}$, or equivalently that

$$
\sum_{\sigma_{\tilde{t}}, \beta_{\tilde{t}}} A\left(\tilde{t}, \sigma_{\tilde{t}}, 67\right) \mathcal{S}\left[\sigma_{\tilde{t}} \mid \beta_{\tilde{t}}\right] \mathcal{C}\left[\tilde{t}, 6, \beta_{\tilde{t}}, 7\right]=\sum_{\sigma_{2} \in \operatorname{perm}\{345\}} A\left(2, \sigma_{2}, 67\right) \mathcal{S}\left[\sigma_{2} \mid 345\right]_{6}
$$

for each $\tilde{t}$. To keep the derivation simple we introduce the following shorthand notation for repeatedly occurring factors

$$
T_{\tilde{t}}\left(\beta_{\tilde{t}}\right)=\sum_{\sigma_{\tilde{t}}} A\left(\tilde{t}, \sigma_{\tilde{t}}, 67\right) \mathcal{S}\left[\sigma_{\tilde{t}} \mid \beta_{\tilde{t}}\right] .
$$

so that for example when $\tilde{t}=5$, equation (A.18) reads

$$
\sum_{\beta_{5} \in \operatorname{perm}\{234\}} T_{5}\left(\beta_{5}\right) \mathcal{C}\left[5,6, \beta_{5}, 7\right]=T_{2}(345) .
$$


Substituting the explicit expressions for translation coefficients $\mathcal{C}_{\mathrm{s}}$, the left hand side of the above equation becomes

$$
\begin{aligned}
& T_{5}(234)+\left(-T_{5}(234)+T_{5}(324)\right) \frac{s_{32}+s_{36}}{s_{32}} \\
& +\left(-T_{5}(243)+T_{5}(423)\right) \frac{s_{42}+S_{46}+s_{43}}{s_{42}} \\
& +\left(T_{5}(234)-T_{5}(324)-T_{5}(423)+T_{5}(432)\right) E[34,5] \\
& +\left(T_{5}(243)-T_{5}(324)+T_{5}(342)-T_{5}(423)\right) E[43,5] .
\end{aligned}
$$

With a little bit more effort, we find that the left hand side of (A.20) boils down to the following linear combination of amplitudes.

$$
\begin{aligned}
& -s_{36} s_{46}\left(s_{56}+s_{54}+s_{53}+s_{52}\right) A(523467) \\
& -s_{36}\left(s_{34}+s_{46}\right)\left(s_{56}+s_{54}+s_{53}+s_{52}\right) A(524367) .
\end{aligned}
$$

On the other hand the right hand side of (A.20) reads

$$
\begin{aligned}
T_{2}(345)= & s_{36} s_{46} s_{56} A(234567)+s_{36} s_{46}\left(s_{46}+s_{56}\right) A(235467) \\
& +s_{36} s_{46}\left(s_{53}+s_{54}+s_{56}\right) A(253467) \\
& +s_{36}\left(s_{43}+s_{46}\right) s_{56} A(243567)+s_{36}\left(s_{43}+s_{46}\right)\left(s_{56}+s_{53}\right) A(245367) \\
& +s_{36}\left(s_{43}+s_{46}\right)\left(s_{53}+s_{54}+s_{56}\right) A(254367),
\end{aligned}
$$

We see that the first line of (A.22) matches the sum of the first three terms of equation (A.23), and similarly the second line of (A.22) matches the sum of the last three terms of (A.23) because of BCJ relation, thereby proving the identity (A.20). The situations when $\tilde{t}=3$ and 4 can be proved in a likewise manner.

Identity (4.18). At 7-points the difference term $\Delta_{n=7}$ splits into four parts, $\Delta_{n=7}=$ $\sum_{t=2,3,4,5} \Delta_{n=7}^{t}$, where

$$
\Delta_{n=7}^{t}=\sum_{\sigma, \beta \in S_{3}} A(t, \sigma, 6,7) \mathcal{S}[\sigma \mid \beta]_{6} J_{t} \tilde{A}(t, 6, \beta, 7)
$$

Substituting the above expressions into equation (A.24) and collecting terms, we find as in the previous examples that terms where angular momentum operate on basis amplitudes $\tilde{A}$ add up to zero because of angular momentum conservation $\sum_{t} J_{t} \tilde{A}(2,6,3,4,5,7)=0$, leaving us with the collection of terms that $J_{t}$ operate on expansion coefficients $\mathcal{C}$, which are functions of kinematic variables. Contributions from the three respective sectors are given by

$$
\begin{aligned}
\Delta_{t=3}= & \left(-T_{3}(245)+T_{3}(425)\right) J_{3}\left(\frac{s_{42}+s_{46}+s_{43}}{s_{42}}\right) \\
& +\left(-T_{3}(254)+T_{3}(524)\right) J_{3}\left(\frac{s_{52}+s_{56}+s_{53}+s_{54}}{s_{52}}\right) \\
& +\left(T_{3}(245)-T_{3}(425)-T_{3}(524)+T_{3}(542)\right) J_{3}(E[45,3]) \\
& +\left(T_{3}(254)-T_{3}(425)+T_{3}(452)-T_{3}(524)\right) J_{3}(E[54,3]),
\end{aligned}
$$




$$
\begin{aligned}
\Delta_{t=4}= & \left(-T_{3}(235)+T_{3}(325)\right) J_{3}\left(\frac{s_{32}+s_{36}}{s_{32}}\right)+\left(-T_{3}(253)+T_{3}(523)\right) J_{3}\left(\frac{s_{52}+s_{56}+s_{53}+s_{54}}{s_{52}}\right) \\
& +\left(T_{3}(235)-T_{3}(325)-T_{3}(523)+T_{3}(532)\right) J_{3}(E[35,4]) \\
& +\left(T_{3}(253)-T_{3}(325)+T_{3}(4352)-T_{3}(523)\right) J_{3}(E[53,4]), \\
\Delta_{t=5}= & \left(-T_{3}(234)+T_{3}(324)\right) J_{3}\left(\frac{s_{32}+s_{36}}{s_{32}}\right)+\left(-T_{3}(243)+T_{3}(423)\right) J_{3}\left(\frac{s_{42}+s_{46}+s_{43}}{s_{42}}\right) \\
& +\left(T_{3}(234)-T_{3}(324)-T_{3}(423)+T_{3}(432)\right) J_{3}(E[34,5]) \\
& +\left(T_{3}(243)-T_{3}(324)+T_{3}(342)-T_{3}(423)\right) J_{3}(E[43,5]),
\end{aligned}
$$

Generically the operation of $J_{t}$ on kinematic variables must fall into one of the following categories:

- $t=3$,

$$
\begin{aligned}
& J_{3 \dot{\alpha} \dot{\beta}} s_{i 6}=\frac{i}{2} \tilde{\lambda}_{3(\dot{\alpha}} \tilde{\lambda}_{i \dot{\beta})}(-) \frac{\langle i 6\rangle\langle 73\rangle}{\langle 76\rangle}, \quad i=2,4,5 \\
& J_{3 \dot{\alpha} \dot{\beta}} s_{i 7}=\frac{i}{2} \tilde{\lambda}_{3(\dot{\alpha}} \tilde{\lambda}_{i \dot{\beta})}(-) \frac{\langle i 7\rangle\langle 63\rangle}{\langle 67\rangle} \\
& J_{3 \dot{\alpha} \dot{\beta}} s_{i 3}=\frac{i}{2} \tilde{\lambda}_{3(\dot{\alpha}} \tilde{\lambda}_{i \dot{\beta})}\langle i 3\rangle \\
& J_{3 \dot{\alpha} \dot{\beta}} s_{i i^{\prime}}=0, \quad i, i^{\prime}=2,4,5
\end{aligned}
$$

- $t=4$,

$$
\begin{aligned}
J_{4 \dot{\alpha} \dot{\beta}} s_{i 6} & =\frac{i}{2} \tilde{\lambda}_{4(\dot{\alpha}} \tilde{\lambda}_{i \dot{\beta})}(-) \frac{\langle i 6\rangle\langle 74\rangle}{\langle 76\rangle}, \quad i=3,4,5 \\
J_{4 \dot{\alpha} \dot{\beta}} s_{i 7} & =\frac{i}{2} \tilde{\lambda}_{4(\dot{\alpha}} \tilde{\lambda}_{i \dot{\beta})}(-) \frac{\langle i 7\rangle\langle 64\rangle}{\langle 67\rangle} \\
J_{4 \dot{\alpha} \dot{\beta}} s_{i 4} & =\frac{i}{2} \tilde{\lambda}_{4(\dot{\alpha}} \tilde{\lambda}_{i \dot{\beta})}\langle i 4\rangle \\
J_{4 \dot{\alpha} \dot{\beta}} s_{i i^{\prime}} & =0, \quad i, i^{\prime}=3,4,5
\end{aligned}
$$

- $t=5$,

$$
\begin{aligned}
J_{5 \dot{\alpha} \dot{\beta}} s_{i 6} & =\frac{i}{2} \tilde{\lambda}_{5(\dot{\alpha}} \tilde{\lambda}_{i \dot{\beta})}(-) \frac{\langle i 6\rangle\langle 75\rangle}{\langle 76\rangle}, \quad i=2,4,5 \\
J_{5 \dot{\alpha} \dot{\beta}} s_{i 7} & =\frac{i}{2} \tilde{\lambda}_{5(\dot{\alpha}} \tilde{\lambda}_{i \dot{\beta})}(-) \frac{\langle i 7\rangle\langle 65\rangle}{\langle 67\rangle} \\
J_{5 \dot{\alpha} \dot{\beta}} s_{i 3} & =\frac{i}{2} \tilde{\lambda}_{5(\dot{\alpha}} \tilde{\lambda}_{i \dot{\beta})}\langle i 5\rangle \\
J_{5 \dot{\alpha} \dot{\beta}} s_{i i^{\prime}} & =0, \quad i, i^{\prime}=2,4,5
\end{aligned}
$$

Suppose if we are interested in checking terms carrying $\tilde{\lambda}_{3(\dot{\alpha}} \tilde{\lambda}_{4 \dot{\beta})}$. Before we commence an explicit calculation, note that because all of the $\mathcal{C}_{\mathrm{S}}$ do not depend explicitly on leg 7 , from the list above such a term can only be produced through $J_{3}\left(s_{46}\right), J_{3}\left(s_{43}\right), J_{4}\left(s_{36}\right)$, 
$J_{4}\left(s_{34}\right)$, which allows us to ignore the $t=5$ sector entirely. Additionally since $s_{34}$ happen to be absent from the $t=4$ translation coefficient $\mathcal{C}$ s, this leaves only $J_{3}\left(s_{46}\right), J_{3}\left(s_{43}\right)$, $J_{4}\left(s_{36}\right)$. Considering the explicit forms given by equations (A.28), (A.29) and (A.30) we further note that (again) because of the absence of the leg 7 dependence in all $\mathcal{C}_{\mathrm{s}}$, the contributions from $J_{3}\left(s_{46}\right), J_{3}\left(s_{43}\right), J_{4}\left(s_{36}\right)$ together can only cancel through Jacobi identity $\langle 43\rangle+\frac{\langle 73\rangle\langle 64\rangle}{\langle 76\rangle}+\frac{\langle 74\rangle\langle 36\rangle}{\langle 76\rangle}=0$. For that to happen, the contribution associated with $J_{3}\left(s_{46}\right)$, $J_{3}\left(s_{43}\right), J_{4}\left(s_{36}\right)$ must be exactly in the ratio $1: 1:-1$, in other words they must add up to

$$
J_{3}\left(s_{46}\right) X+J_{3}\left(s_{43}\right) X+J_{4}\left(s_{36}\right)(-X)=0
$$

for some factor $X$. In the following discussion we shall see that indeed this is the case.

First we note that it is relatively easy to confirm that the ratio between the contributions from $J_{3}\left(s_{46}\right)$ and $J_{3}\left(s_{43}\right)$ is $1: 1$. This can be seen by observing that the kinematic factors $s_{46}$ and $s_{43}$ always show up together through the combination $s_{46}+s_{43}$ in all of the translation coefficients $\mathcal{C}$ in the $t=3$ sector (see equations from (A.5) to (A.6) as well as (A.25)). The only part of the argument that requires explicit calculation is the ratio between $J_{3}\left(s_{46}\right)$ and $J_{4}\left(s_{36}\right)$. For the purpose of discussion let us tentatively call them respectively as $X$ and $Y$. From equation (A.25) and the definition of $E[45,3]$ and $E[54,3]$, the contribution associated with $J_{3}\left(s_{46}\right)$ reads

$$
\begin{aligned}
X= & \frac{1}{s_{42} s_{52}\left(s_{45}+s_{42}+s_{52}\right)}\left[s_{52}\left(s_{45}+s_{42}+s_{52}\right)\left(-T_{3}(245)+T_{3}(425)\right)\right. \\
& +s_{52}\left(s_{52}+s_{56}+s_{53}+s_{54}\right)\left(T_{3}(245)-T_{3}(425)-T_{3}(524)+T_{3}(542)\right) \\
& \left.+s_{42}\left(s_{52}+s_{56}+s_{53}+s_{54}\right)\left(T_{3}(254)-T_{3}(425)+T_{3}(452)-T_{3}(524)\right)\right] \\
= & {\left[s_{36} s_{56} A(4,2,3,5,6,7)+s_{36}\left(s_{35}+s_{56}\right) A(4,2,5,3,6,7)\right.} \\
& -s_{56}\left(s_{25}+s_{26}+s_{56}+s_{35}+s_{45}\right) A(4,3,2,5,6,7)+s_{26}\left(s_{35}+s_{45}\right) A(4,3,5,2,6,7) \\
& \left.-s_{36} s_{45} A(4,5,2,3,6,7)+s_{26} s_{45} A(4,5,3,2,6,7)\right]
\end{aligned}
$$

and similarly,

$$
\begin{aligned}
Y= & \frac{1}{s_{32} s_{52}\left(s_{52}+s_{32}+s_{35}\right)}\left[s_{52}\left(s_{52}+s_{32}+s_{35}\right)\left(-T_{4}(235)+T_{4}(325)\right)\right. \\
& +s_{32}\left(s_{52}+s_{53}+s_{54}+s_{56}\right)\left(T_{4}(253)-T_{4}(325)+T_{4}(352)-T_{4}(523)\right) \\
& \left.+s_{52}\left(s_{52}+s_{53}+s_{54}+s_{56}\right)\left(T_{4}(235)-T_{4}(325)-T_{4}(523)+T_{4}(532)\right)\right] \\
= & s_{36} s_{56} A(4,2,3,5,6,7)+s_{36}\left(s_{35}+s_{56}\right) A(4,2,5,3,6,7) \\
& -s_{56}\left(s_{25}+s_{26}+s_{56}+s_{35}+s_{45}\right) A(4,3,2,5,6,7)+s_{26}\left(s_{35}+s_{45}\right) A(4,3,5,2,6,7) \\
& \left.-s_{36} s_{45} A(4,5,2,3,6,7)+s_{26} s_{45} A(4,5,3,2,6,7)\right]
\end{aligned}
$$

Now that we have the explicit formulas of the $J_{3}\left(s_{46}\right)$ and $J_{4}\left(s_{36}\right)$ term contributions, it is evident from (A.33) and (A.34) that they are related by an exchange of legs 3 and 4 , $Y=\left.X\right|_{3 \leftrightarrow 4}$. Therefore to prove $X=-Y$ it suffices to show that $Y$ is antisymmetric with respect to indices 3 and 4 . This antisymmetric structure will become manifest after some nontrivial manipulations, which we perform in the following. 
First of all note that BCJ relation allows us to write

$$
\begin{aligned}
& s_{36} s_{56} A(4,2,3,5,6,7)+s_{36}\left(s_{35}+s_{56}\right) A(4,2,5,3,6,7) \\
= & -s_{36}\left(s_{52}+s_{53}+s_{56}\right) A(4,5,2,3,6,7)-s_{36}\left(s_{52}+s_{53}+s_{56}+s_{54}\right) A(4,2,3,6,7,5)
\end{aligned}
$$

and

$$
\begin{aligned}
& s_{26}\left(s_{35}+s_{45}\right) A(4,3,5,2,6,7)+s_{26} s_{45} A(4,5,3,2,6,7) \\
= & -s_{26}\left(s_{35}+s_{45}+s_{25}\right) A(4,3,2,5,6,7)-s_{26}\left(s_{35}+s_{45}+s_{25}+s_{65}\right) A(4,3,2,6,5,7) .
\end{aligned}
$$

Plugging the above two identities into the expression for $Y$, we have

$$
\begin{aligned}
Y= & -s_{36}\left(s_{52}+s_{53}+s_{56}+s_{54}\right)[A(4,5,2,3,6,7)+A(4,2,3,6,7,5)] \\
& -\left[s_{26}\left(s_{35}+s_{45}+s_{25}\right)+s_{56}\left(s_{25}+s_{26}+s_{56}+s_{35}+s_{45}\right)\right] A(4,3,2,5,6,7) \\
& -s_{26}\left(s_{35}+s_{45}+s_{25}+s_{65}\right) A(4,3,2,6,5,7) \\
= & -\left(s_{52}+s_{53}+s_{56}+s_{54}\right) \\
& \times\left[s_{36} A(4,5,2,3,6,7)+s_{36} A(4,2,3,6,7,5)+\left(s_{26}+s_{56}\right) A(4,3,2,5,6,7)+s_{26} A(4,3,2,6,5,7)\right] \\
= & s_{57}\left[s_{36} A(4,5,2,3,6,7)+s_{36} A(4,2,3,6,7,5)+\left(s_{26}+s_{56}\right) A(4,3,2,5,6,7)+s_{26} A(4,3,2,6,5,7)\right]
\end{aligned}
$$

Further using BCJ relation identifies the sum of last two terms above with

$$
\begin{aligned}
& \left(s_{26}+s_{56}\right) A(4,3,2,5,6,7)+s_{26} A(4,3,2,6,5,7) \\
& =-\left(s_{26}+s_{56}+s_{76}\right) A(4,3,2,5,7,6)-\left(s_{26}+s_{56}+s_{76}+s_{46}\right) A(4,6,3,2,5,7) \\
& =\left(s_{36}+s_{46}\right) A(4,3,2,5,7,6)+s_{36} A(4,6,3,2,5,7)
\end{aligned}
$$

Therefore Y simplifies as

$$
\begin{aligned}
Y= & s_{57} s_{36}[A(4,5,2,3,6,7)+A(4,2,3,6,7,5)+A(4,3,2,5,7,6)+A(4,6,3,2,5,7)] \\
& +s_{57} s_{46} A(4,3,2,5,7,6) \\
= & s_{57}\left[s_{46} A(4,3,2,5,7,6)-s_{36} A(3,4,2,5,7,6)\right]
\end{aligned}
$$

where we used U(1) decoupling identity to substitute the summation in the first line with a single amplitude. The final simplified formula of $Y$ is manifestly antisymmetric under the exchange of indices 3 and 4 , and we conclude that $X+Y=0$ as claimed.

Open Access. This article is distributed under the terms of the Creative Commons Attribution License (CC-BY 4.0), which permits any use, distribution and reproduction in any medium, provided the original author(s) and source are credited.

\section{References}

[1] F.E. Low, Scattering of light of very low frequency by systems of spin 1/2, Phys. Rev. 96 (1954) 1428 [INSPIRE]. 
[2] F.E. Low, Bremsstrahlung of very low-energy quanta in elementary particle collisions, Phys. Rev. 110 (1958) 974 [INSPIRE].

[3] M. Gell-Mann and M.L. Goldberger, Scattering of low-energy photons by particles of spin 1/2, Phys. Rev. 96 (1954) 1433 [inSPIRE].

[4] S. Saito, Low-energy theorem for Compton scattering, Phys. Rev. 184 (1969) 1894 [INSPIRE].

[5] S. Weinberg, Photons and Gravitons in s Matrix Theory: Derivation of Charge Conservation and Equality of Gravitational and Inertial Mass, Phys. Rev. 135 (1964) B1049.

[6] S. Weinberg, Infrared photons and gravitons, Phys. Rev. 140 (1965) B516 [InSPIRE].

[7] A. Strominger, On BMS Invariance of Gravitational Scattering, JHEP 07 (2014) 152 [arXiv: 1312.2229] [INSPIRE].

[8] T. He, V. Lysov, P. Mitra and A. Strominger, BMS supertranslations and Weinberg's soft graviton theorem, arXiv:1401.7026 [INSPIRE].

[9] D. Kapec, V. Lysov, S. Pasterski and A. Strominger, Semiclassical Virasoro symmetry of the quantum gravity $\mathcal{S}$-matrix, JHEP 08 (2014) 058 [arXiv:1406.3312] [INSPIRE].

[10] R. Britto, F. Cachazo and B. Feng, New recursion relations for tree amplitudes of gluons, Nucl. Phys. B $\mathbf{7 1 5}$ (2005) 499 [hep-th/0412308] [INSPIRE].

[11] R. Britto, F. Cachazo, B. Feng and E. Witten, Direct proof of tree-level recursion relation in Yang-Mills theory, Phys. Rev. Lett. 94 (2005) 181602 [hep-th/0501052] [INSPIRE].

[12] F. Cachazo and A. Strominger, Evidence for a New Soft Graviton Theorem, arXiv:1404. 4091 [INSPIRE].

[13] Z. Bern, L.J. Dixon, M. Perelstein and J.S. Rozowsky, Multileg one loop gravity amplitudes from gauge theory, Nucl. Phys. B 546 (1999) 423 [hep-th/9811140] [INSPIRE].

[14] E. Laenen, G. Stavenga and C.D. White, Path integral approach to eikonal and next-to-eikonal exponentiation, JHEP 03 (2009) 054 [arXiv: 0811.2067] [INSPIRE].

[15] E. Laenen, L. Magnea, G. Stavenga and C.D. White, Next-to-eikonal corrections to soft gluon radiation: a diagrammatic approach, JHEP 01 (2011) 141 [arXiv:1010.1860] [INSPIRE].

[16] C.D. White, Factorization Properties of Soft Graviton Amplitudes, JHEP 05 (2011) 060 [arXiv: 1103.2981] [INSPIRE].

[17] E. Casali, Soft sub-leading divergences in Yang-Mills amplitudes, arXiv:1404.5551 [INSPIRE].

[18] J. Broedel, M. de Leeuw, J. Plefka and M. Rosso, Constraining subleading soft gluon and graviton theorems, Phys. Rev. D 90 (2014) 065024 [arXiv:1406.6574] [INSPIRE].

[19] Z. Bern, S. Davies, P. Di Vecchia and J. Nohle, Low-Energy Behavior of Gluons and Gravitons from Gauge Invariance, Phys. Rev. D 90 (2014) 084035 [arXiv:1406.6987] [INSPIRE].

[20] C.D. White, Diagrammatic insights into next-to-soft corrections, Phys. Lett. B 737 (2014) 216 [arXiv: 1406.7184] [INSPIRE].

[21] A.J. Larkoski, Conformal Invariance of the Subleading Soft Theorem in Gauge Theory, Phys. Rev. D 90 (2014) 087701 [arXiv: 1405.2346] [InSPIRE].

[22] B.U.W. Schwab and A. Volovich, Subleading soft theorem in arbitrary dimension from scattering equations, Phys. Rev. Lett. 113 (2014) 101601 [arXiv:1404.7749] [InSPIRE]. 
[23] N. Afkhami-Jeddi, Soft Graviton Theorem in Arbitrary Dimensions, arXiv:1405.3533 [INSPIRE].

[24] M. Zlotnikov, Sub-sub-leading soft-graviton theorem in arbitrary dimension, JHEP 10 (2014) 148 [arXiv: 1407.5936] [INSPIRE].

[25] C. Kalousios and F. Rojas, Next to subleading soft-graviton theorem in arbitrary dimensions, arXiv: 1407.5982 [INSPIRE].

[26] Z. Bern, S. Davies and J. Nohle, On Loop Corrections to Subleading Soft Behavior of Gluons and Gravitons, Phys. Rev. D 90 (2014) 085015 [arXiv:1405.1015] [InSPIRE].

[27] S. He, Y.-t. Huang and C. Wen, Loop Corrections to Soft Theorems in Gauge Theories and Gravity, arXiv:1405.1410 [INSPIRE].

[28] F. Cachazo and E.Y. Yuan, Are Soft Theorems Renormalized?, arXiv:1405.3413 [INSPIRE].

[29] B.U.W. Schwab, Subleading Soft Factor for String Disk Amplitudes, JHEP 08 (2014) 062 [arXiv: 1406.4172] [INSPIRE].

[30] M. Bianchi, S. He, Y.-t. Huang and C. Wen, More on Soft Theorems: Trees, Loops and Strings, arXiv: 1406.5155 [INSPIRE].

[31] T. Adamo, E. Casali and D. Skinner, Perturbative gravity at null infinity, Class. Quant. Grav. 31 (2014) 225008 [arXiv: 1405.5122] [INSPIRE].

[32] Y. Geyer, A.E. Lipstein and L. Mason, Ambitwistor strings at null infinity and subleading soft limits, arXiv:1406.1462 [INSPIRE].

[33] Z. Bern, J.J.M. Carrasco and H. Johansson, New Relations for Gauge-Theory Amplitudes, Phys. Rev. D 78 (2008) 085011 [arXiv: 0805.3993] [INSPIRE].

[34] F. Cachazo, S. He and E.Y. Yuan, Scattering of Massless Particles in Arbitrary Dimensions, Phys. Rev. Lett. 113 (2014) 171601 [arXiv:1307.2199] [INSPIRE].

[35] F. Cachazo, S. He and E.Y. Yuan, Scattering of Massless Particles: Scalars, Gluons and Gravitons, JHEP 07 (2014) 033 [arXiv:1309.0885] [INSPIRE].

[36] H. Kawai, D.C. Lewellen and S.H.H. Tye, A Relation Between Tree Amplitudes of Closed and Open Strings, Nucl. Phys. B 269 (1986) 1 [InSPIRE].

[37] N.E.J. Bjerrum-Bohr, P.H. Damgaard, B. Feng and T. Sondergaard, Gravity and Yang-Mills Amplitude Relations, Phys. Rev. D 82 (2010) 107702 [arXiv: 1005.4367] [inSPIRE].

[38] N.E.J. Bjerrum-Bohr, P.H. Damgaard, B. Feng and T. Sondergaard, Proof of Gravity and Yang-Mills Amplitude Relations, JHEP 09 (2010) 067 [arXiv:1007.3111] [INSPIRE].

[39] N.E.J. Bjerrum-Bohr, P.H. Damgaard, T. Sondergaard and P. Vanhove, The Momentum Kernel of Gauge and Gravity Theories, JHEP 01 (2011) 001 [arXiv:1010.3933] [INSPIRE].

[40] F. Cachazo and Y. Geyer, A 'Twistor String' Inspired Formula For Tree-Level Scattering Amplitudes in $N=8$ SUGRA, arXiv:1206.6511 [INSPIRE].

[41] F. Cachazo and D. Skinner, Gravity from Rational Curves in Twistor Space, Phys. Rev. Lett. 110 (2013) 161301 [arXiv:1207.0741] [INSPIRE].

[42] F. Cachazo, Resultants and Gravity Amplitudes, arXiv:1301.3970 [INSPIRE]. 